\title{
Building a Smart EM Environment - AI-Enhanced Aperiodic Micro-Scale Design of Passive EM Skins
}

\author{
G. Oliveri, ${ }^{(1)}$ Senior Member, IEEE, F. Zardi, ${ }^{(1)}$ P. Rocca, ${ }^{(1)(2)}$ Senior Member, IEEE, M. Salucci, ${ }^{(1)}$ \\ Member, IEEE, and A. Massa, ${ }^{(1)(3)(4)}$ Fellow, IEEE
}

(1) CNIT - "University of Trento" ELEDIA Research Unit

Via Sommarive 9, 38123 Trento - Italy

E-mail: \{giacomo.oliveri, francesco.zardi, paolo.rocca, marco.salucci, andrea.massa\} @unitn.it

Website: www.eledia.org/eledia-unitn

(2)ELEDIA Research Center (ELEDIA@XIDIAN - Xidian University)

P.O. Box 191, No.2 South Tabai Road, 710071 Xi'an, Shaanxi Province - China

E-mail: paolo.rocca@xidian.edu.cn

Website: www.eledia.org/eledia-xidian

(3)ELEDIA Research Center (ELEDIA@UESTC - UESTC)

School of Electronic Engineering, Chengdu 611731 - China

E-mail: andrea.massa@uestc.edu.cn

Website: www.eledia.org/eledia-uestc

(4)ELEDIA Research Center (ELEDIA@TSINGHUA - Tsinghua University)

30 Shuangqing Rd, 100084 Haidian, Beijing - China

E-mail: andrea.massa@tsinghua.edu.cn

Website: www.eledia.org/eledia-tsinghua

This work has been submitted to the IEEE for possible publication. Copyright may be transferred without notice, after which this version may no longer be accessible. 


\title{
Building a Smart EM Environment - AI-Enhanced Aperiodic Micro-Scale Design of Passive EM Skins
}

G. Oliveri, F. Zardi, P. Rocca, M. Salucci, and A. Massa

\begin{abstract}
An innovative process for the design of static passive smart skins (SPSSs) is proposed to take into account, within the synthesis, the electromagnetic $(E M)$ interactions due to their finite (macro-level) size and aperiodic (micro-scale) layouts. Such an approach leverages on the combination of an inverse source (IS) formulation, to define the SPSS surface currents, and of an instance of the System-by-Design paradigm, to synthesize the unit cell (UC) descriptors suitable for supporting these currents. As for this latter step, an enhanced Artificial Intelligence (IA)-based digital twin $(D T)$ is built to efficiently and reliably predict the relationships among the $U C \mathrm{~s}$ and the non-uniform coupling effects arising when the $U C \mathrm{~s}$ are irregularly assembled to build the corresponding SPSS. Towards this end and unlike state-of-the-art approaches, an aperiodic finite small-scale model of the SPSS is derived to generate the training database for the $D T$ implementation. A set of representative numerical experiments, dealing with different radiation objectives and smart skin apertures, is reported to assess the reliability of the conceived design process and to illustrate the radiation features of the resulting layouts, validated with accurate full-wave simulations, as well.
\end{abstract}

Key words: Smart Skins; EM Holography; Next-Generation Communications; Iterative Projection Method; System-by-Design; Metasurfaces; Metamaterials. 


\section{Introduction and Rationale}

The Smart Electromagnetic Environment (SEE) vision is at the core of a revolutionary approach currently emerging in the design and the implementation of next generation wireless cellular systems [1]-[9]. The academic and industrial interest in such a transformative paradigm is motivated by the unprecedented SEE potentialities in terms of blind spot mitigation, coverage improvement, and electromagnetic (EM) propagation control enabled by the opportunistic exploitation of the wireless power already available within the propagation scenario [2][7][10]. Moreover, a minimization of the power consumption of the wireless infrastructure and a reduction of the overall EM pollution are also assured thanks to the improved wireless efficiency without the need of installing new base stations [1]-[9].

Within this framework, static passive smart skins (SPSSs) have been conceived to guarantee the minimum impact in terms of fabrication, installation, maintenance, costs, and power consumption [7][11]. A SPSS is an artificial passive structure that "manipulates" (i.e., reflection/focusing) the incident power radiated by one or more base stations towards a desired coverage region [7][11]. Towards this end, SPSS implementations usually leverage on the capabilities of passive modulated thin metasurfaces to control the EM reflection, thanks to their micro-scale physical structure [11][12], rather than using active elements such as diodes, varactors, phase shifters, or amplifiers [12][13]. Therefore, there are not running costs after the installation and no electrical nor processing power is required to operate, thus making SPSSs particularly attractive for an inexpensive and fast deployment in large-scale scenarios [11]. Indeed, despite the technological simplicity and the relatively low cost implementation, SPSSs can yield an excellent EM propagation control as already evidenced in several and different "Surface EM" applications including lenses, beam splitters, wave polarizers, and reflect/transmit arrays [12]. However, the design of practically feasible SPSSs in SEE must fulfil very strict and contrasting requirements often not occurring in standard Surface EM problems [11]. On the one hand, the use of multi-layer/complex shapes or expensive materials in the unit cells (UCs) of the SPSS is prevented for costs and weight reasons. On the other hand, there is the need of high-performance in terms of both pattern shaping and independence on the polarization of the incident field. To address such challenges, a customization of the System-by-Design (SbD) paradigm [4][14]- 
[16] has been recently proposed in [11]. By formulating the complex multi-scale $E M$ design problem at hand according to the Generalized Sheet Transition Condition (GSTC) technique [12][17]-[19], the approach in [11] combines a phase-only inverse source $(I S)$ method, to find the reference surface currents induced on the structure with the Iterative Projection Technique (IPT) [20], together with an integration between a global optimization algorithm and a UC Digital Twin (UC-DT) to determine the descriptors of the SPSS that supports the IPT-synthesized currents [11]. In order to significantly speed-up the generation of the training set for learning the $U C-D T$, the $U C$ response is modeled by assuming local periodicity conditions [11] as usually done in the literature on surface electromagnetics [12] [14] [15] [21]-[23]. Unfortunately, the aperiodicity and the edge effects of finite layouts introduce a degree of approximation which is very difficult to compensate in the final full-scale SPSS. Such an issue is particularly challenging when dealing with electrically large apertures since the optimization of the finite layout becomes computationally unmanageable. This paper then proposes an innovative approach to face with these challenges by extending and generalizing the method conceived in [11]. More in detail, the SPSS synthesis is still split into two-steps, but unlike [11], the UC-DT of the second step (i.e., the definition of the micro-scale descriptors of the $U C$ so that the arising modulated metasurface supports the reference currents synthesized at the first $I S$ step) avoids periodic assumptions and it predicts the behavior of each $U C$ by taking into account a finite set of neighbouring cells as well as different locations of the same $U C$ within the so-called small-scale model. The local susceptibility tensor of this latter is then computed with a fullwave commercial solver [24] and afterwards exploited in an Artificial Intelligence (AI)-driven learning process to build the "Local $U C-D T$ ".

To the best of the authors' knowledge, the main innovative contributions of this work include (i) the introduction of a Local UC-DT for the reliable design of effective large-scale SPSSs by taking into account the local aperiodicity and the edge effects of the finite structure, (ii) an efficient integration of such a $D T$ within the $S b D$-based $S P S S$ synthesis process, and (iii) the full-wave modeling/simulation of the arising SPSS layouts to carefully assess their performance in realistic operative scenarios.

The outline of the paper is as follows. The design problem at hand is formulated in Sect. 
2, while the proposed synthesis process is detailed in Sect. 3, A selected set of numerical examples is reported and the synthesis outcomes are assessed with full-wave simulations in realistic operative conditions (Sect. 4). Finally, come conclusions follow (Sect. 5).

\section{SPSS Problem Formulation}

Let us consider the SEE scenario in Fig. 1 where a SPSS of $P \times Q U C$ s, arranged on the $x y$ plane according to a regular lattice with spacings $\Delta x$ and $\Delta y$, is illuminated by a plane wave of $T E / T M$ complex components $E_{T E}^{i n c} / E_{T M}^{i n c}$ that impinges from the angular direction $\left(\theta^{i n c}, \varphi^{i n c}\right)$. The design of the SPSS can be stated as follows

SPSS Design Problem - Given $\left(\theta^{i n c}, \varphi^{i n c}\right), E_{T E}^{i n c}$, and $E_{T M}^{i n c}$, find $\mathbf{G}$ such that $\mathcal{O}[\mathbf{F}(\mathbf{r} ; \mathbf{G})]$ is minimized

where $\mathbf{G}$ is the vector of the descriptors of the SPSS (i.e., the set of $P \times Q U C \mathrm{~s}),(\mathbf{G} \triangleq$ $\left.\left\{\mathbf{g}_{p q} ; p=1, \ldots, P, q=1, \ldots, Q\right\}\right)$, whose $D$-sized $(p, q)$-th $(p=1, \ldots, P ; q=1, \ldots, Q)$ entry, $\mathbf{g}_{p q} \triangleq\left\{g_{p q}^{(d)} ; d=1, \ldots, D\right\}$, lists the geometrical/physical micro-scale parameters of the corresponding $U C$. Moreover, $\mathbf{F}(\mathbf{r} ; \mathbf{G})$ is the electric field reflected by the SPSS in a far-field point with local coordinates $\mathbf{r}=(x, y, z)$, while $\mathcal{O}[\mathbf{F}(\mathbf{r} ; \mathbf{G})]$ is the implicit form of the macro-scale radiation objectives set on the far-field pattern, $\mathbf{F}(\mathbf{r} ; \mathbf{G})$, and whose explicit expression is defined case-by-case according to the specific applicative context.

Regardless of $\mathcal{O}[\mathbf{F}(\mathbf{r})]$, the solution of the SPSS Design Problem requires a reliable approach to compute $\mathbf{F}(\mathbf{r} ; \mathbf{G})$ starting from $\left(\theta^{i n c}, \varphi^{i n c}\right), E_{T E}^{i n c}, E_{T M}^{i n c}$, and $\mathbf{G}$. In principle, such a task may be accomplished by modeling the entire SPSS layout and computing the solution with a full-wave numerical solver. This strategy is practically prevented because of the unfeasible computational costs related to the need of performing an expensive computation of $\mathbf{F}(\mathbf{r} ; \mathbf{G})$ for each guess configuration of $\mathbf{G}$. On the other hand, it is worth pointing out that a careful modelling of the SPSS allows the designer to exploit the intrinsic non-uniqueness of the underlying $I S$ problem (i.e., the deduction of the SPSS surface currents from the radiated far-field pattern) that gives a greater flexibility in the synthesis of wave manipulation devices [25]. 
By taking into account these considerations, a different approach is adopted by exploiting the concept of equivalent surface currents withing the GSTC framework [11][12]. More in detail, the far field pattern reflected by the SPSS is defined as $\mathbf{F}(\mathbf{r} ; \mathbf{G}) \triangleq \mathcal{L}\left[\mathbf{J}^{\text {tot }}(\mathbf{r} ; \mathbf{G})\right], \mathcal{L}[\cdot]$ being the far-field Green's operator, and it computed as [11] [26]

$$
\mathbf{F}(\mathbf{r} ; \mathbf{G})=\frac{j k_{0}}{4 \pi} \frac{\exp \left(-j k_{0}|\mathbf{r}|\right)}{|\mathbf{r}|} \int_{\Xi}\left\{\mathbf{J}^{t o t}(\widetilde{\mathbf{r}} ; \mathbf{G}) \exp \left(j k_{0} \widehat{\mathbf{r}} \cdot \widetilde{\mathbf{r}}\right)\right\} \mathrm{d} \widetilde{\mathbf{r}}
$$

where $\widehat{\mathbf{r}} \triangleq \frac{\mathbf{r}}{|\mathbf{r}|}, k_{0}$ is the free-space wavenumber and $\Xi$ is the SPSS surface aperture, while $\mathbf{J}^{\text {tot }}$ is the surface equivalent source induced on $\Xi()$ and computed as the superposition of the electric, $\mathbf{J}^{e}$, and the magnetic, $\mathbf{J}^{m}$, effective current components

$$
\mathbf{J}^{t o t}(\mathbf{r} ; \mathbf{G})=\widehat{\mathbf{r}} \times\left[\eta_{0} \widehat{\mathbf{r}} \times \mathbf{J}^{e}(\mathbf{r} ; \mathbf{G})+\mathbf{J}^{m}(\mathbf{r} ; \mathbf{G})\right]
$$

$\eta_{0}$ being the free-space impedance $\left(\eta_{0} \triangleq \sqrt{\frac{\mu_{0}}{\varepsilon_{0}}}, \varepsilon_{0}\right.$ and $\mu_{0}$ being the free-space permittivity and permeability, respectively).

According to the GSTC technique [12][19], the current components $\mathbf{J}^{e}$ and $\mathbf{J}^{m}$ are functions of the electric, $\mathbf{S}^{e}(\mathbf{r} ; \mathbf{G})$, and the magnetic, $\mathbf{S}^{m}(\mathbf{r} ; \mathbf{G})$, polarization surface densities so that $\mathbf{J}^{e}(\mathbf{r} ; \mathbf{G})=j \omega \mathbf{S}_{t}^{e}(\mathbf{r} ; \mathbf{G})-\widehat{\mathbf{n}} \times \nabla_{t} S_{n}^{m}(\mathbf{r} ; \mathbf{G})$ and $\mathbf{J}^{m}(\mathbf{r} ; \mathbf{G})=j \omega \mu_{0} \mathbf{S}_{t}^{m}(\mathbf{r} ; \mathbf{G})+\frac{1}{\varepsilon_{0}} \widehat{\mathbf{n}} \times \nabla_{t} S_{n}^{e}(\mathbf{r} ; \mathbf{G})$, $\widehat{\mathbf{n}}$ being the normal to the smart skin surface $\Xi$, while $\mathbf{S}_{t}^{e / m}(\mathbf{r} ; \mathbf{G}) \triangleq \mathbf{S}^{e / m}(\mathbf{r} ; \mathbf{G}) \times \widehat{\mathbf{n}}$ and $S_{n}^{e / m}(\mathbf{r} ; \mathbf{G}) \triangleq \mathbf{S}^{e / m}(\mathbf{r} ; \mathbf{G}) \cdot \widehat{\mathbf{n}}$. Moreover, for (sufficiently) symmetric $U C \mathbf{s}, \mathbf{S}^{e}$ and $\mathbf{S}^{m}$ are given by

$$
\mathbf{S}^{e}(\mathbf{r} ; \mathbf{G})=\sum_{p=1}^{P} \sum_{q=1}^{Q}\left[\varepsilon_{0} \overline{\bar{\psi}}_{p q}^{e}(\mathbf{G}) \cdot \mathbf{E}_{p q}^{a v e}(\mathbf{G})\right] \Psi^{p q}(\mathbf{r})
$$

and

$$
\mathbf{S}^{m}(\mathbf{r} ; \mathbf{G})=\sum_{p=1}^{P} \sum_{q=1}^{Q}\left[\overline{\bar{\psi}}_{p q}^{m}(\mathbf{G}) \cdot \mathbf{H}_{p q}^{a v e}(\mathbf{G})\right] \Psi^{p q}(\mathbf{r})
$$

where $\Psi^{p q}(\mathbf{r}) \triangleq\left\{\right.$ if $\mathbf{r} \in \Xi_{p q}, 0$ if $\left.\mathbf{r} \notin \Xi_{p q}\right\}, \Xi_{p q}$ being the support of the $(p, q)$-th $(p=$ $1, \ldots, P ; q=1, \ldots, Q) U C$ so that $\Xi=\sum_{p=1}^{P} \sum_{q=1}^{Q} \Xi_{p q}$.

The computation of (3) and (4) only requires to determine the electric/magnetic local surface susceptibility diagonal tensors $\overline{\bar{\psi}}_{p q}^{e / m}(\mathbf{G})$ of the $(p, q)$-th $(p=1, \ldots, P ; q=1, \ldots, Q) U C$ since the surface averaged fields $\Phi_{p q}^{a v e}(\mathbf{G})(\boldsymbol{\Phi}=\{\mathbf{E}, \mathbf{H}\})$ are given by [11] [12] 


$$
\boldsymbol{\Phi}_{p q}^{a v e}(\mathbf{G}) \triangleq \frac{1}{2 \times \Delta x \times \Delta y} \int_{\Xi_{p q}}\left\{\mathbf{1}+\overline{\bar{R}}\left[\overline{\bar{\psi}}_{p q}^{e / m}(\mathbf{G})\right]\right\} \cdot \boldsymbol{\Phi}^{i n c}(\mathbf{r}) d \mathbf{r} \quad \boldsymbol{\Phi}=\{\mathbf{E}, \mathbf{H}\}
$$

where the incident electric,

$$
\mathbf{E}^{i n c}(\mathbf{r}) \triangleq\left(E_{T E}^{i n c} \widehat{\mathbf{e}}_{T E}+E_{T M}^{i n c} \widehat{\mathbf{e}}_{T M}\right) \exp \left(-j \mathbf{k}^{i n c} \cdot \mathbf{r}\right)
$$

and magnetic,

$$
\mathbf{H}^{i n c}(\mathbf{r}) \triangleq \frac{1}{\eta_{0} k_{0}} \mathbf{k}^{i n c} \times \mathbf{E}^{i n c}(\mathbf{r})
$$

fields are known quantities, $\mathbf{k}^{i n c}$ and $\widehat{\mathbf{e}}_{T E / T M}$ being the incident wave vector $\left(\mathbf{k}^{i n c} \triangleq-k_{0}\right.$ $\left.\left[\sin \left(\theta^{i n c}\right) \cos \left(\varphi^{i n c}\right) \widehat{\mathbf{x}}+\sin \left(\theta^{i n c}\right) \sin \left(\varphi^{i n c}\right) \widehat{\mathbf{y}}+\cos \left(\theta^{i n c}\right) \widehat{\mathbf{z}}\right]\right)$ and the TE/TM mode unit vectors $\left(\widehat{\mathbf{e}}_{T E}=\frac{\mathbf{k}^{i n c} \times \widehat{\mathbf{n}}}{\left|\mathbf{k}^{i n c} \times \widehat{\mathbf{n}}\right|} ; \widehat{\mathbf{e}}_{T M}=\frac{\widehat{\mathbf{e}}_{T E} \times \mathbf{k}^{i n c}}{\left|\widehat{\mathbf{e}}_{T E} \times \mathbf{k}^{i n c}\right|}\right)$, respectively, while $\mathbf{1}$ is a diagonal unitary tensor, and $\overline{\bar{R}}\left[\overline{\bar{\psi}}_{p q}^{e / m}(\mathbf{G})\right] \triangleq R^{T E, T E}\left[\overline{\bar{\psi}}_{p q}^{e / m}(\mathbf{G})\right] \widehat{\mathbf{e}}_{T E} \widehat{\mathbf{e}}_{T E}+R^{T E, T M}\left[\overline{\bar{\psi}}_{p q}^{e / m}(\mathbf{G})\right] \widehat{\mathbf{e}}_{T E} \widehat{\mathbf{e}}_{T M}+$ $R^{T M, T E}\left[\overline{\bar{\psi}}_{p q}^{e / m}(\mathbf{G})\right] \widehat{\mathbf{e}}_{T M} \widehat{\mathbf{e}}_{T E}+R^{T M, T M}\left[\overline{\bar{\psi}}_{p q}^{e / m}(\mathbf{G})\right] \widehat{\mathbf{e}}_{T M} \widehat{\mathbf{e}}_{T M}$ is the local reflection tensor, which can be derived from $\overline{\bar{\psi}}_{p q}^{e / m}(\mathbf{G})[12]$.

It is worthwhile to point out that here, unlike [11], the electric/magnetic local surface susceptibility diagonal tensors $\overline{\bar{\psi}}_{p q}^{e / m}$ of the $(p, q)$-th $(p=1, \ldots, P ; q=1, \ldots, Q) U C$ depends on the whole finite structure of the $\operatorname{SPSS}\left[\right.$ i.e., $\overline{\bar{\psi}}_{p q}^{e / m}=\overline{\bar{\psi}}_{p q}^{e / m}(\mathbf{G})$ ] instead of on the $D$ descriptors of the same $(p, q)$-th $U C$ [i.e., $\left.\overline{\bar{\psi}}_{p q}^{e / m}=\overline{\bar{\psi}}_{p q}^{e / m}\left(\mathbf{g}_{p q}\right)\right]$. The interested readers should also notice that such an $I S$-based formulation of the SPSS problem [i.e., equation (1) through (3) and (4) with the intermediate step (2) for the computation of the induced surface current, $\left.\mathbf{J}^{\text {tot }}(\mathbf{r} ; \mathbf{G})\right]$ allows one to exploit the multiplicity of the induced currents [25] to identify the most proper (i.e., physicallyadmissible and easy-to-build) SPSS layout for the scenario/objectives at hand. On the other hand, it is evident the multi-scale nature of the SPSS synthesis problem since the fulfilment of the macro-scale objectives, $\mathcal{O}[\mathbf{F}(\mathbf{r} ; \mathbf{G})]$, is obtained by optimizing the micro-scale descriptors of the UCs, G. Last but not least, the design of a SPSS intrinsically features complexity and high-dimensionality since the number of degrees-of-freedom ( $D o F \mathrm{~s}), \mathcal{N} \triangleq P \times Q \times D$, rapidly increases with the SPSS aperture, $\Xi$, and the number of descriptors of the $U C, D$. 


\section{SPSS Synthesis Procedure}

According to the mathematical formulation in Sect. 2 and referring to (1), the SPSS design is split into two steps [11], as usually done also in reflectarray engineering [15][28], concerned with a macro-scale constrained $I S$ problem followed by a local current matching step. More specifically, the SPSS layout is synthesized by first computing the optimal reference current, $\mathbf{J}_{o p t}^{t o t}$, that radiates a far field pattern, $\mathbf{F}_{\text {opt }}(\mathbf{r}) \triangleq \mathcal{L}\left\{\mathbf{J}_{\text {opt }}^{\text {tot }}(\mathbf{r})\right\}$, minimizing $\mathcal{O}\left[\mathbf{F}_{\text {opt }}(\mathbf{r})\right]$, then finding the optimal setup of the SPSS descriptors, $\mathbf{G}_{o p t}$, such that

$$
\mathbf{G}_{\text {opt }}=\arg \left[\min _{\mathbf{G}}\{\Delta(\mathbf{G})\}\right]
$$

where $\Delta(\mathbf{G})$ is the surface current mismatch $\left(\Delta(\mathbf{G}) \triangleq\left\|\mathbf{J}^{t o t}(\mathbf{r} ; \mathbf{G})-\mathbf{J}_{\text {opt }}^{\text {tot }}(\mathbf{r})\right\|_{\Xi}^{2},\|\cdot\|_{\Xi}\right.$ being the $\ell_{2}$-norm over $\Xi$ given by $\|\cdot\|_{\Xi} \triangleq \sqrt{\left.\int_{\Xi}|\cdot|^{2} \mathrm{~d} \mathbf{r}\right)}$.

The first step is carried out as in [11] by choosing an IPT-based approach [20] to find the optimal SPSS currents. Towards this end, the "pattern" feasibility space $\mathcal{W}\{\mathbf{F}(\mathbf{r})\}$ and the "current" feasibility space $\mathcal{W}\left\{\mathbf{J}^{t o t}(\mathbf{r})\right\}$ as well as the mutual projection operators are defined. The former, $\mathcal{W}\{\mathbf{F}(\mathbf{r})\}$, is cast into the following mask-matching form

$$
\mathcal{W}\{\mathbf{F}(\mathbf{r})\} \triangleq\left\{\mathbf{F}(\mathbf{r}):|\mathbf{F}(\mathbf{r})|^{2} \geq \mathcal{M}(\mathbf{r}) ; \mathbf{r} \in \Theta\right\}
$$

where $\mathcal{M}(\mathbf{r})$ is the user-defined lower footprint power mask in the coverage region $\Theta$. Moreover, let us describe the feasibility space of the "current" as $\mathcal{W}\left\{\mathbf{J}^{t o t}(\mathbf{r})\right\} \triangleq\left\{\mathbf{J}^{t o t}(\mathbf{r}): \mathbf{J}^{t o t}(\mathbf{r})\right.$ $=C \exp [j \chi(\mathbf{r})] ; \mathbf{r} \in \Xi\}, C$ and $\chi(\mathbf{r})$ being the magnitude constant and the locally-controlled phase distribution of the surface current, respectively.

The IPT synthesis process iteratively updates the $i$-th $(i=1, \ldots, I)$ guess current, $\mathbf{J}_{i}^{\text {tot }}$ ( $i$ being the iteration index), starting from a random distribution, $\mathbf{J}_{0}^{\text {tot }}$, and alternatively computing the $i$-th $(i=1, \ldots, I)$ "projected pattern", $\widetilde{\mathbf{F}}_{i}\left(\mathbf{F}_{i}(\mathbf{r}) \triangleq \mathcal{L}\left[\mathbf{J}_{i}^{\text {tot }}(\mathbf{r})\right]\right)$,

$$
\widetilde{\mathbf{F}}_{i}(\mathbf{r})= \begin{cases}\sqrt{\mathcal{M}(\mathbf{r})} & \text { if }\left|\mathbf{F}_{i}(\mathbf{r})\right|^{2} \leq \mathcal{M}(\mathbf{r}) \\ \mathbf{F}_{i}(\mathbf{r}) & \text { otherwise }\end{cases}
$$


and the "projected current" at the next $(i+1)$-th iteration

$$
\mathbf{J}_{i+1}^{t o t}(\mathbf{r})=\frac{\mathbf{J}_{i}^{M N}(\mathbf{r})}{\left\|\mathbf{J}_{i}^{M N}(\mathbf{r})\right\|}
$$

where $\mathbf{J}_{i}^{M N}$ is the minimum norm solution of the integral equation (1) (i.e., $\mathbf{J}_{i}^{M N}(\mathbf{r}) \triangleq \mathcal{L}_{M N}^{-1}$ $\left\{\widetilde{\mathbf{F}}_{i}(\mathbf{r})\right\}$ ), which is determined with the truncated singular value decomposition [25].

The process is stopped when either the maximum number of IPT iterations has been reached (i.e., $i=I)$ or the value of the pattern matching index, $\Gamma_{i}(i<I)$, given by

$$
\Gamma_{i}=\frac{\left\|\widetilde{\mathbf{F}}_{i}(\mathbf{r})-\mathbf{F}_{i}(\mathbf{r})\right\|_{\Theta}}{\left\|\mathbf{F}_{i}(\mathbf{r})\right\|_{\Theta}}
$$

is smaller than a user-defined convergence threshold, $\gamma$, and the estimate of the surface current distribution is outputted by setting $\mathbf{J}_{\text {opt }}^{\text {tot }}(\mathbf{r})=\mathbf{J}_{i}^{\text {tot }}(\mathbf{r}), \mathbf{r} \in \Xi$.

As for the second step towards the SPSS synthesis, solving (8) to find $\mathbf{G}_{\text {opt }}$ is a computationally challenging optimization task owing to the number and the heterogeneity of the DoFs and the cost function at hand, $\Delta(\mathbf{G})$. According to the guidelines in the global optimization literature (see for instance [29][4] and the reference therein), an iterative $S b D$-based strategy is applied by generating a succession of $N$ trial sets, $\left\{\mathcal{P}^{(n)} ; n=1, \ldots, N\right\}$ ( $n$ being the iteration index during the optimization), with the Particle Swarm mechanism [29] and computing the current mismatch, $\Delta\left(\left.\mathbf{G}^{a}\right|^{(n)}\right)$, for each $a$-th $(a=1, \ldots, A)$ guess SPSS layout of each $n$-th $(n=1, \ldots, N)$ iteration/swarm being $\mathcal{P}^{(n)}=\left\{\left.\mathbf{G}^{a}\right|^{(n)} ; a=1, \ldots, A\right\}$, while $A$ is the swarm size. The calculation of $\Delta\left(\left.\mathrm{G}^{a}\right|^{(n)}\right)$ requires the knowledge of the surface current $\mathbf{J}^{\text {tot }}$ in correspondence with the $a$-th $(a=1, \ldots, A)$ layout of the $n$-th $(n=1, \ldots, N)$ swarm, $\left.\mathbf{G}^{a}\right|^{(n)}[$ i.e., $\left.\mathbf{J}^{t o t}\left(\left.\mathbf{G}^{a}\right|^{(n)}\right)\right]$, which is accomplished once the electric and the magnetic polarization surface densities, $\mathbf{S}^{e / m}\left(\mathbf{r} ;\left.\mathbf{G}^{a}\right|^{(n)}\right)$, are determined through (3) and (4). Towards this end, the key task is to deduce the local surface susceptibility tensors of the $(p, q)$-th $(p=1, \ldots, P ; q=1, \ldots, Q)$ $U C, \overline{\bar{\psi}}_{p q}^{e / m}$, but depending on the whole SPSS arrangement [i.e., $\overline{\bar{\psi}}_{p q}^{e / m}=\overline{\bar{\psi}}_{p q}^{e / m}\left(\left.\mathbf{G}^{a}\right|^{(n)}\right)$ ]. Because of the computational costs, the use of a full-wave simulator is impossible since it would imply the expensive EM modelling of the behavior of $P \times Q \times A \times N$ full-size SPSSs. To reduce the computational burden, the EM response of the SPSS has been emulated with a $U C$ - 
$D T$, which has been trained by exploiting a single-cell full-wave model under the assumption of periodic boundary conditions [11]. However, neglecting the aperiodicity and the edge effects of the actual finite-size SPSS introduces a non-negligible degree of approximation. In order to avoid such a drawback and unlike [11], a Local UC-DT is defined to substitute the actual $\overline{\bar{\psi}}_{p q}^{e / m}(\mathbf{G})$ with its surrogate $\overline{\bar{\zeta}}_{p q}^{e / m}(\mathbf{G})$ learned offline from a small-scale model of the SPSS. More specifically, such a Local UC-DT is implemented according to the following procedure:

- Small-Scale SPSS Modeling - An aperiodic $P^{\prime} \times Q^{\prime}$ (with $P^{\prime} \ll P, Q^{\prime} \ll Q$ ) smallscale SPSS layout, which is described by the reduced vector of descriptors $G^{\prime}\left(G^{\prime} \triangleq\right.$ $\left.\left\{\mathbf{g}_{p^{\prime} q^{\prime}} ; p^{\prime}=1, \ldots, P^{\prime} ; q^{\prime}=1, \ldots, Q^{\prime}\right\}\right)$ whose $\left(p^{\prime}, q^{\prime}\right)$-th entry is still of size $D$ (i.e., $\mathbf{g}_{p^{\prime} q^{\prime}} \triangleq$ $\left\{g_{p^{\prime} q^{\prime}}^{(d)} ; d=1, \ldots, D\right\}$, is built;

- Small-Scale SPSS Training Set Definition - A representative set of $B$ variations of the $P^{\prime} \times Q^{\prime} D$-size small-scale descriptors is considered to derive $B$ small-scale SPSS layouts, $\left\{\mathbf{G}_{b}^{\prime} ; b=1, \ldots, B\right\}$, then the behavior of each $b$-th $(b=1, \ldots, B)$ layout is full-wave simulated to predict its $E M$ behavior by extracting the corresponding susceptibility tensors, $\left\{\overline{\bar{\psi}}_{p^{\prime} q^{\prime}}^{e / m}\left(\mathbf{G}_{b}^{\prime}\right) ; p^{\prime}=1, \ldots, P^{\prime} ; q^{\prime}=1, \ldots, Q^{\prime}\right\}$, from the local scattering parameters [12];

- Small-Scale SPSS AI-based Surrogate Model Creation - The AI-based Local UC-DT surrogate model $\overline{\bar{\zeta}}_{p^{\prime} q^{\prime}}^{e / m}\left(\mathbf{G}^{\prime}\right)\left(p^{\prime}=1, \ldots, P^{\prime} ; q^{\prime}=1, \ldots, Q^{\prime}\right)$ is created starting from the small-scale $B$-size SPSS training set, $\left\{\left[\mathbf{G}_{b}^{\prime}, \overline{\bar{\psi}}_{p^{\prime} q^{\prime}}^{e / m}\left(\mathbf{G}_{\mathbf{b}}^{\prime}\right)\right] ; b=1, . ., B\right\}$, by means of a statistical learning approach based on the Ordinary Kriging $(O K)$ method [15][23]. More in detail, the value of $\overline{\bar{\zeta}}_{p^{\prime} q^{\prime}}^{e / m}\left(\mathbf{G}^{\prime}\right)\left(p^{\prime}=1, \ldots, P^{\prime} ; q^{\prime}=1, \ldots, Q^{\prime}\right)$ is predicted as follows

$$
\begin{aligned}
& \overline{\bar{\zeta}}_{p^{\prime} q^{\prime}}^{e / m}\left(\mathbf{G}^{\prime}\right)=\wp_{3}^{\text {reg }}\left\{\psi_{p^{\prime} q^{\prime}}^{e / m}\left(\mathbf{G}_{b}^{\prime}\right) ; \mathbf{c}\right\} \Im_{3 \times 3}^{\text {unit }}+\left[\wp_{B}^{\text {corr }}\left\{\mathbf{G}^{\prime} ; \mathbf{c}\right\}\right]^{\dagger}\left[\Im_{B \times B}^{\text {corr }}\left\{\mathbf{G}_{b}^{\prime} ; \mathbf{c}\right\}\right]^{-1} \\
& \times\left(\Im_{B \times 3}^{\text {train }}\left\{\psi_{p^{\prime} q^{\prime}}^{e / m}\left(\mathbf{G}_{b}^{\prime}\right)\right\}-\wp_{B}^{\text {unit }} \wp_{3}^{(4)}\left\{\psi_{p^{\prime} q^{\prime}}^{e / m}\left(\mathbf{G}_{b}^{\prime}\right) ; \mathbf{c}\right\}\left\{\psi_{p^{\prime} q^{\prime}}^{e / m}\left(\mathbf{G}_{b}^{\prime}\right) ; \mathbf{c}\right\}\right) \Im_{3 \times 3}^{\text {unit }}
\end{aligned}
$$

where

$$
\begin{gathered}
\wp_{3}^{\text {reg }}\left\{\psi_{p^{\prime} q^{\prime}}^{e / m}\left(\mathbf{G}_{b}^{\prime}\right) ; \mathbf{c}\right\} \triangleq\left(\left[\wp_{B}^{\text {unit }}\right]^{\dagger}\left[\Im_{B \times B}^{\text {corr }}\left\{\mathbf{G}_{b}^{\prime} ; \mathbf{c}\right\}\right]^{-1} \wp_{B}^{\text {unit }}\right)^{-1} \\
{\left[\wp_{B}^{\text {unit }}\right]^{\dagger}\left[\Im_{B \times B}^{\text {corr }}\left\{\mathbf{G}_{b}^{\prime} ; \mathbf{c}\right\}\right]^{-1} \Im_{B \times 3}^{\text {train }}\left\{\psi_{p^{\prime} q^{\prime}}^{e / m}\left(\mathbf{G}_{b}^{\prime}\right)\right\}}
\end{gathered}
$$

is the regression parameter matrix. $\wp_{B}^{\text {corr }}\left\{\mathbf{G}^{\prime} ; \mathbf{c}\right\}$ is the $B$-size exponential correlation

\footnotetext{
${ }^{(1)}$ For notation simplicity, the symbol $\Im_{u \times v}$ is used to indicate a matrix of $u$ rows and $v$ columns, while $\wp_{u}$
} 
vector $\left(\wp_{B}^{\operatorname{corr}}\left\{\mathbf{G}^{\prime} ; \mathbf{c}\right\} \triangleq\left\{Z_{b}\left(\mathbf{G}^{\prime} ; \mathbf{c}\right)=\exp \left(-\sum_{p^{\prime}=1}^{P^{\prime}} \sum_{q^{\prime}=1}^{Q^{\prime}} \sum_{d=1}^{D} c_{n} \mid g_{p^{\prime} q^{\prime}}^{(d)}-g_{p^{\prime} q^{\prime}}^{(d)}\right\rfloor_{b} \mid\right)\right.$; $b=1, \ldots, B\}$ being $\left.n=p^{\prime}+P^{\prime} \times\left(q^{\prime}-1\right)+P^{\prime} \times Q^{\prime} \times(d-1)\right), \Im_{B \times B}^{\text {corr }}\left\{\mathbf{G}_{b}^{\prime} ; \mathbf{c}\right\}$ is a $B \times B$ matrix whose $b$-th $(b=1, \ldots, B)$ column is equal to $\wp_{B}^{\text {corr }}\left\{\mathbf{G}^{\prime} ; \mathbf{c}\right\}$, while $\mathbf{c} \triangleq$ $\left\{c_{n} ; n=1, \ldots, \mathcal{N}\right\}$ is the set of $\mathcal{N}$ control parameters, which are automatically optimized during the $O K$ self-tuning [23]. Moreover, $\Im_{B \times 3}^{\text {train }}\left\{\psi_{p^{\prime} q^{\prime}}^{e / m}\left(\mathbf{G}_{b}^{\prime}\right)\right\}$ is the training-set matrix whose $(w, b)$-th $(w=x, y, z ; b=1, \ldots, B)$ entry is equal to the (ww)-th component of the local surface susceptibility diagonal tensor of the $\left(p^{\prime}, q^{\prime}\right)$-th $U C$ of the $b$-th smallscale SPSS layout, $\mathbf{G}_{b}^{\prime}$, (i.e., $\Im_{B \times 3}^{\text {train }}\left\{\psi_{p^{\prime} q^{\prime}}^{e / m}\left(\mathbf{G}_{b}^{\prime}\right)\right\} \triangleq\left\{\left.\psi_{p^{\prime} q^{\prime}}^{e / m}\left(\mathbf{G}_{b}^{\prime}\right)\right|_{w w} ; w=x, y, z ; b=\right.$ $1, \ldots, B\})$ being [11][12]

$$
\left.\overline{\bar{\psi}}_{p^{\prime} q^{\prime}}^{e / m}\left(\mathbf{G}_{b}^{\prime}\right) \triangleq \sum_{w=x, y, z} \psi_{p^{\prime} q^{\prime}}^{e / m}\left(\mathbf{G}_{b}^{\prime}\right)\right|_{w w} \widehat{w} \widehat{w}
$$

while $\wp_{B}^{\text {unit }}$ is a unitary column vector of length $B$ and $\cdot^{\dagger}$ stands for the transpose operator;

- Small-Scale to Full-Scale SPSS Prediction Mapping - The actual local surface susceptibility tensors of the full-size $\operatorname{SPSS},\left\{\overline{\bar{\psi}}_{p q}^{e / m}(\mathbf{G}) ; p=1, \ldots, P ; q=1, \ldots, Q\right\}$, are then estimated by deriving $\left\{\overline{\bar{\zeta}}_{p q}^{e / m}(\mathbf{G}) ; p=1, \ldots, P ; q=1, \ldots, Q\right\}$ from the $O K$ small-scale SPSS predictions, $\left\{\overline{\bar{\zeta}}_{p^{\prime} q^{\prime}}^{e / m}\left(\mathbf{G}^{\prime}\right) ; p^{\prime}=1, \ldots, P^{\prime} ; q^{\prime}=1, \ldots, Q^{\prime}\right\}$ according to the mapping scheme pictorially illustrated in Fig. 2 and performed according to the rules in Appendix.

Once the Local UC-DT has been implemented offline and it is available, the SbD-based SPSS synthesis process can be efficiently executed, even multiple, times without recurring to additional full-wave simulations of either a part or the whole of the $U C$ s arrangement. Moreover, no further training is required even if the synthesis objectives $\mathcal{O}[\mathbf{F}(\mathbf{r} ; \mathbf{G})]$ are changed after the Local UC-DT was created. Those features assure to the arising design method high-scalability and re-usability properties.

identifies a vector of $u$ rows. 


\section{Numerical Results}

In this section, a selected set of examples from a wide numerical analysis is reported to illustrate the proposed $A I$-enhanced aperiodic micro-scale design procedure for the synthesis of SPSSs working in real scenarios as well as to assess the reliability of the synthesized layouts in operative conditions through full-wave simulations. More specifically, the numerical validation has been carried out by considering various supports of the SPSS and different radiation objectives, $\mathcal{O}[\mathbf{F}(\mathbf{r} ; \mathbf{G})]$. As for this latter, the Pencil Coverage and the Shaped Coverage cases have been chosen since as largely representative of several real applications (2). In the Pencil Coverage case, the power reflected by the SPSS is focused towards a desired (anomalous non-Snell) target direction, $\left(\theta^{T}, \varphi^{T}\right)$, which means setting $\mathcal{O}[\mathbf{F}(\mathbf{r} ; \mathbf{G})]=\mathcal{O}^{\text {pen }}[\mathbf{F}(\mathbf{r} ; \mathbf{G})]\left[\mathcal{O}^{\text {pen }}[\mathbf{F}(\mathbf{r} ; \mathbf{G})] \triangleq\right.$ $\frac{1}{\left|\mathbf{F}\left(r^{T}, \theta^{T}, \varphi^{T} ; \mathbf{G}\right)\right|^{2}}$ where $r^{T}$ is an arbitrary (far-field) distance, while $|\cdot|$ stands for the vector magnitude) and $\Theta=\left(r^{T}, \theta^{T}, \varphi^{T}\right)$ in (9). Otherwise, the SPSS of the Shaped Coverage case maximizes the reflected power in the user-defined footprint/coverage area $\Theta$ (Fig. 1), thus the synthesis objective is set to $\mathcal{O}[\mathbf{F}(\mathbf{r} ; \mathbf{G})]=\mathcal{O}^{\text {sha }}[\mathbf{F}(\mathbf{r} ; \mathbf{G})]\left(\mathcal{O}^{\text {sha }}[\mathbf{F}(\mathbf{r} ; \mathbf{G})] \triangleq \frac{1}{\int_{\Omega}|\mathbf{F}(\mathbf{r}) ; \mathbf{G}|^{2} d \mathbf{r}}\right)$. Finally, $\mathcal{M}(\mathbf{r})=0[\mathrm{~dB}]$ has been set in both cases.

Concerning the benchmark SEE scenario, a base station has been assumed to illuminate the SPSS at $f=3.5[\mathrm{GHz}]$ (i.e., sub-6GHz $n 78$ band [30]) with a plane wave featuring circular polarization (i.e., $E_{T E}^{i n c}=1$ and $E_{T M}^{i n c}=j$ ) and impinging from broadside (i.e., $\left(\theta^{i n c}, \varphi^{i n c}\right)=$ $(0,0)[\operatorname{deg}] \rightarrow \widehat{\mathbf{e}}_{T E}=\widehat{\mathbf{y}}$ and $\left.\widehat{\mathbf{e}}_{T M}=\widehat{\mathbf{x}}\right)$. Moreover, the calibration parameters of the synthesis procedure have been set following the guidelines in [11][15]: $B=2 \times 10^{4}, \gamma=10^{-4}, I=10^{3}$, $A=10$, and $N=10^{4}$. Finally, the full-wave simulations have been carried out with Ansys HFSS [24].

In order to illustrate the proposed SPSS design method, let start with the offline process of building the Local UC-DT, which does not depend on the synthesis objective (i.e., $\mathcal{O}[\mathbf{F}(\mathbf{r} ; \mathbf{G})]$ ), to be defined for the (second) step of the SbD-based SPSS layout synthesis and kept in all numerical tests. Accordingly, a $U C$ consisting of a metallic square patch of side $\ell(D=1)$ printed on a Rogers RT/duroid 5870 laminate with thickness $\tau=3.175 \times 10^{-3}$ [m] [Fig. 3(a)] has been selected as the benchmark SPSS element. It can be noticed [Figs. 3(c)-3(d)] that

\footnotetext{
${ }^{(2)}$ It is worth remarking that the solution process is independent on $\mathcal{O}[\mathbf{F}(\mathbf{r} ; \mathbf{G})]$.
} 
the phase coverage of such a $U C$, which presents a structural resonance centered around $\ell=$ $2.5 \times 10^{-2}[\mathrm{~m}]$, is not complete [i.e., $\approx 330[\mathrm{deg}]$ at $f=3.5 \mathrm{GHz}-$ Fig. $\left.3(c)\right]$ as a consequence of the simple shape and the single-layer design. The choice of a basic/elementary $U C$ has been made to assess the effectiveness of the proposed approach without the bias of a highperformance $U C$. Then, a small-scale SPSS layout with $P^{\prime} \times Q^{\prime}=5 \times 5 U C \mathrm{~s}\left[g_{p^{\prime} q^{\prime}}^{(d)}\right]_{d=1}=\ell_{p^{\prime} q^{\prime}}$, Fig. 3(b)], arranged on a uniform lattice with periodicity $\Delta x=\Delta y=4.28 \times 10^{-2}[\mathrm{~m}]$, has been defined ("Small-Scale SPSS Modeling") and simulated in a full-wave fashion taking into account the copper thickness $\left(\nu=35 \times 10^{-6}[\mathrm{~m}]\right)$, as well. The $B$-sized $O K$ training dataset has been built by varying the $P^{\prime} \times Q^{\prime}$ patch sides [i.e., $\ell_{p^{\prime} q^{\prime}}\left(p^{\prime}=1, \ldots, P^{\prime} ; q^{\prime}=1, \ldots, Q^{\prime}\right)$ ] of the small-scale SPSS layout ("Small-Scale SPSS Training Set Definition”). Starting from this training set, an $O K$-based surrogate of the Small-Scale SPSS model has been built by deriving $\overline{\bar{\zeta}}_{p^{\prime} q^{\prime}}^{e / m}\left(\mathbf{G}^{\prime}\right)\left(p^{\prime}=1, \ldots, P^{\prime} ; q^{\prime}=1, \ldots, Q^{\prime}\right)$. Some insights on the accuracy of the arising predictor can be inferred by the plots of the phase [Fig. 3(c)] and of the magnitude [Fig. 3(d)] of the average "TE-TE" reflection coefficient of a representative element [i.e., the central one located at $\left.\left(p^{\prime}, q^{\prime}\right)=\left(\frac{P^{\prime}+1}{2}, \frac{Q^{\prime}+1}{2}\right)\right]$ of the small-scale SPSS layout

$$
\widetilde{R}_{p^{\prime} q^{\prime}}^{T E, T E}=\frac{1}{B} \sum_{b=1}^{B} R^{T E, T E}\left[\overline{\bar{\psi}}_{p^{\prime} q^{\prime}}^{e / m}\left(\mathbf{G}_{b}^{\prime}\right)\right]_{p^{\prime}=\frac{P^{\prime}+1}{2}}^{q^{\prime}=\frac{Q^{\prime}+1}{2}}
$$

As it can be observed, the predicted curve (i.e., $\widetilde{R}_{p^{\prime} q^{\prime}}^{T E, T E}=\frac{1}{B} \sum_{b=1}^{B} R^{T E, T E}\left[\overline{\bar{\zeta}}_{p^{\prime} q^{\prime}}^{e / m}\left(\mathbf{G}_{b}^{\prime}\right)\right]_{p^{\prime}=\frac{P^{\prime}+1}{2}}^{q^{\prime}=\frac{Q^{\prime}+1}{2}}$ ) faithfully matches the actual one passing throughout the "training" samples as expected from the $O K$ theory.

Once the "Small-Scale SPSS AI-based Surrogate Model" has been created, it is then possible to deal with the $S E E$ problem at hand, which is user-defined by setting the macro-scale radiation objectives (i.e., $\mathcal{O}[\mathbf{F}(\mathbf{r} ; \mathbf{G})]$ ) as well as the SPSS support and position in the global reference system of coordinates $\left(x_{g l o b}, y_{g l o b}, z_{g l o b}\right)$ (Fig. 1).

The first test case of the numerical assessment is concerned with the synthesis of a SPSS installed at $H=5[\mathrm{~m}]$ over the ground (Fig. 1) with $P \times Q=30 \times 30 U C$ s (i.e., $\Xi \approx 1.28 \times 1.28$ $\left.\left[\mathrm{m}^{2}\right]\right)$ to yield a Pencil Coverage with focusing direction $\left(\theta^{T}, \varphi^{T}\right)=(50,-8)$ [deg], which corresponds to a footprint spot at $x_{g l o b}=-35.7[\mathrm{~m}], y_{g l o b}=30.14[\mathrm{~m}]$. By following the 
procedure described in Sect. 3, the first step has been carried out with the IPT [Fig. 4(a)] to compute the optimal reference current, $\mathbf{J}_{\text {opt }}^{\text {tot }}[$ Fig. $4(b)(3)]$, that radiates the far field pattern $\mathbf{F}_{\text {opt }}(\mathbf{r}) \triangleq \mathcal{L}\left\{\mathbf{J}_{\text {opt }}^{\text {tot }}(\mathbf{r})\right\}\left[\right.$ Fig. 4(c)] by minimizing $\mathcal{O}^{\text {pen }}\left[\mathbf{F}_{\text {opt }}(\mathbf{r})\right]$. Figure 4(a) shows that there is a quick reduction of the value of the pattern matching index $\Gamma_{i}(12)(i=1, \ldots, I)$, which confirms the effectiveness of the IPT when applied to fulfil macro-scale objectives (i.e., reflection performance) by optimizing micro-scale $D o F$ s (i.e., the surface currents). It is also worth pointing out that the synthesized current distribution only depends on the project targets and not on the $U C$ geometry, hence the same surface current can be kept also varying the $U C$ materials, shapes, layers, etc .... In the second step of the SPSS synthesis, the layout in Fig. 5(a) has been derived from the $S b D$-driven optimization of the $U C$ descriptors to match the reference current profile in Fig. 4(b). As a matter of fact, the arising surface current, $\mathbf{J}_{N}^{\text {tot }}[$ Fig. $5(b)]$, turns out to be very close to the reference one, the same similarity being present between the radiated far-field patterns in the $(u, v)$-domain $(u \triangleq \sin \theta \cos \varphi$ and $v \triangleq \sin \theta \sin \varphi)$ [Fig. 5(c) vs. Fig. 4(c)]. Both outcomes confirm the effectiveness of the optimization process that allows the designer to analytically synthesize a large SPSS (i.e., $30 \times 30 U C s$ ) with a careful control of the resulting reflection/focusing properties. As for this latter item, it turns out that there is the need of a non-uniform arrangement of the $U C$ s [Fig. 5(a)] to afford a "double anomalous" (i.e., $\theta^{T} \neq \theta^{\text {Snell }}$ and $\varphi^{T} \neq \varphi^{\text {Snell }}$ being $\left.\theta^{\text {Snell }}=\varphi^{\text {Snell }}=0[\mathrm{deg}]\right)$ reflection of the incident beam. In order to assess the reliability of the synthesis process as well as the effectiveness of the arising design, the SPSS layout has been also HFSS-modeled and the results have been compared in terms of surface currents (i.e., the target of the second step) [Fig. 6(a) vs. Fig. 5(b)] and far-field reflected power patterns [Fig. $6(b)$ vs. Fig. $5(c)$ ] as well as footprint patterns (i.e., the objective of the whole synthesis process) [Fig. 7(b) vs. Fig. 7(a)]. As expected, the plot of the full-wave simulated pattern [Fig. 6(b)] confirms that the synthesized SPSS is able to focus the reflected beam along the desired anomalous direction [i.e., $\left(u^{T}, v^{T}\right)=\left(7.58 \times 10^{-1},-1.06 \times 10^{-1}\right)$ being $u^{T}=\sin \theta^{T} \cos \varphi^{T}$ and $v^{T}=\sin \theta^{T} \sin \varphi^{T}$ ]. Moreover, the HFSS plot indirectly proves the accuracy of the SPSS surrogate since the far-field distributions are very similar [Fig. $6(b)$ vs. Fig. 5(c)] within the whole visible domain except for a minor secondary lobe located at

\footnotetext{
${ }^{(3)}$ For symmetry reasons, the two components of the surface current are identical. Thus, only the $x$-component, $J_{x}^{t o t}(\mathbf{r})$, will be shown.
} 
$u \in[-0.5,-0.4], v \in[-0.3,-0.2]$ [Fig. 6(b)] possibly caused by the higher-order modes arising in the SPSS UCs patches and not taken into account in Sect. 2, The reliability of the designed SPSS is further confirmed by the analytically-computed [Fig. 7(a)] and HFSSsimulated [Fig. 7(b)] footprint power densities analyzed in an area of extension $120 \times 60\left[\mathrm{~m}^{2}\right]$ in front of the smart skin. Both indicate that the peak of the reflected power is maximum within the coverage spot centered at $x_{g l o b}=-35.7[\mathrm{~m}]$ and $y_{g l o b}=30.14[\mathrm{~m}]$ with close values of the directivity index (i.e., $\xi^{p e n}=32.52[\mathrm{~dB}][$ Fig. $7(a)]$ vs. $\xi_{H F S S}^{p e n}=31.91[\mathrm{~dB}][$ Fig. $7(b)]$ being $\xi^{p e n} \triangleq \frac{4 \pi r^{2}|\mathbf{F}(\mathbf{r} ; \mathbf{G})|^{2}}{\int_{0}^{2 \pi} \int_{0}^{\pi}|\mathbf{F}(\mathbf{r} ; \mathbf{G})|^{2} r^{2} \sin (\theta) \mathrm{d} \theta \mathrm{d} \varphi}$ the average directivity in the footprint region for the SEE pencil coverage). Moreover, despite the approximations of the analytic approach (Sect. 2) in modeling the surface currents on $\Xi$, there is a good agreement between the current distributions [Fig. 6(a) vs. Fig. 5(b)] and the surface averaged fields (5), $\left\{\mathbf{E}_{p q}^{\text {ave }}(\mathbf{G}) ; p=1, \ldots, P ; q=1, \ldots, Q\right\}$, [Fig. 7(c) vs. Fig. 7(d)]. This further assesses the effectiveness of the "Small-Scale to Full-Scale SPSS Prediction Mapping“ step in predicting the actual local surface susceptibility tensors of the full-size $S P S S,\left\{\overline{\bar{\psi}}_{p q}^{e / m}(\mathbf{G}) ; p=1, \ldots, P ; q=1, \ldots, Q\right\}$, both $\mathbf{J}^{\text {tot }}(\mathbf{r} ; \mathbf{G})$ and $\mathbf{E}_{p q}^{a v e}(\mathbf{G})$ being related to $\overline{\bar{\psi}}_{p q}^{e / m}(\mathbf{G})$ through (2)-(4) and (5), respectively.

The synthesis of a larger $P \times Q=50 \times 50$ layout (i.e., $\Xi \approx 2.14 \times 2.14\left[\mathrm{~m}^{2}\right]$ ) has been performed next to analyse the method robustness when dealing with higher dimensionalities of the optimization problem, $\mathcal{N}$ being proportional to $P \times Q$, as well as the dependence of the focalization features on the size of the SPSS support. Let us take a look to the $S b D$-synthesized layout in Fig. 8(a). Analogously to the $P=Q=30$ case [Fig. 5(a)], once again the UCs are non-uniform and the patterned surface appears (similarly) irregular as actually expected since the same anomalous coherent reflection of the previous test case is required-and-obtained here [Fig. 8(b)] even though by exploiting a wider aperture. The larger SPSS support implies that the majority of the reflected power along the same desired direction $\left(u^{T}=7.58 \times 10^{-1}\right.$ and $\left.v=-1.06 \times 10^{-1}\right)$ is focused in a narrower beam [Fig. 8(b) vs. Fig. 5(c)] and a more confined coverage footprint [Fig. 8(c) vs. Fig. 7(a)]. Quantitatively, the increase of the SPSS area $\left[\frac{\Xi_{P=Q=50}}{\Xi_{P=Q=30}} \approx 2.77 \rightarrow \delta \Xi=4.42[\mathrm{~dB}]\right.$ - Fig. 8(a) vs. Fig. 5(a)] corresponds to a proportional improvement of the focusing efficiency $\left(\delta \xi^{p e n} \approx 4.5[\mathrm{~dB}]\right)$ being $\left.\xi^{p e n}\right\rfloor_{P=Q=50}=36.96[\mathrm{~dB}]$ [Fig. 8(c)] and $\left.\xi^{p e n}\right\rfloor_{P=Q=30}=32.52[\mathrm{~dB}][$ Fig. $\left.7(a)]\right)$. Such a result $\left(\delta \Xi \approx \delta \xi^{p e n}\right)$ can provide 
useful guidelines on how to size and design the SPSSs for obtaining a desired (selective/broad) coverage in a SEE scenario.

The same conclusions drawn in the first example on the reliability of the synthesis results hold true also for this wider SPSS as confirmed by the comparisons with the HFSS simulations, at micro-scale, of the spatial distributions of both the surface current, $\mathbf{J}^{\text {tot }}(\mathbf{r} ; \mathbf{G})$ [Fig. 9(c) vs. Fig. 8(d)], and the averaged electric field, $\mathbf{E}_{p q}^{a v e}(\mathbf{G})[$ Fig. $9(d)$ vs. Fig. 8(e)], while at macroscale, of the radiated patterns [Fig. $9(a)$ vs. Fig. $8(b)$ ] and footprints [Fig. $9(b)$ vs. Fig. $8(c)]$. For instance, it turns out that $\xi_{H F S S}^{p e n}=36.08[\mathrm{~dB}] \approx \xi^{p e n}=36.96[\mathrm{~dB}]$ so that the rule $\delta \Xi \approx \delta \xi_{H F S S}^{p e n}$ is verified also here.

But what about more complex footprints? The design of SPSSs for shaped coverages is thus discussed by considering two different applicative scenarios, each with a different setup of the pattern-mask region $\Theta$ in (9), while keeping the same SPSS aperture $(P \times Q=50 \times 50)$. More specifically, the former ("Two-Squares Footprint" scenario) mimics the realistic case where the SPSS is requested to afford two separate and asymmetric beams that focus the power in very narrow, but shaped, regions, which emulate two small town-squares in a urban environment. Numerically, $\Theta$ consists of two regions of $20 \times 10\left[\mathrm{~m}^{2}\right]$ centered at $\left(x_{\text {glob }}^{(1)}, y_{\text {glob }}^{(1)}\right)=(-30,15)$ $[\mathrm{m}]$ and $\left(x_{\text {glob }}^{(2)}, y_{\text {glob }}^{(2)}\right)=(28,17)[\mathrm{m}]$, respectively. Otherwise, the coverage region of the "StreetSquare Footprint" case maps a $10 \times 120\left[\mathrm{~m}^{2}\right]$ street centered at $\left(x_{\text {glob }}^{(1)}, y_{\text {glob }}^{(1)}\right)=(-35,80)[\mathrm{m}]$ that opens on a square of size $30 \times 30\left[\mathrm{~m}^{2}\right]$ located at $x_{\text {glob }}^{(2)}=-y_{\text {glob }}^{(2)}=-45[\mathrm{~m}]$. From a methodological viewpoint, such a test case is devoted to assess the potentialities of the SPSS in focusing the power in a region characterized by both complex contours and very low grazing angles, which are almost parallel to the ground surface, due to both the street position and length $\left(L_{\text {street }}=140[\mathrm{~m}]\right)$ and the SPSS height above the ground $(H=5[\mathrm{~m}])$.

The layouts of the synthesized SPSSs [Fig. 10(a) - "Two-Squares Footprint"; Fig. 10(b) "Street-Square Footprint"] are still non-uniform, but less regular than in the "Pencil Coverage" case [Fig. 5(a) and Fig. 8(a)] owing to the complexity of the shaped-beam. As a consequence, the "periodicity" of the spatial behavior of the surface currents in Fig. $8(d)$ is completely lost [Figs. 10(c)-10(d)]. On the other hand, the analytical implementation based on the Local UC$D T$ (Sect. 2) is still very reliable, as confirmed by the full-wave simulations, at both micro- 
[Figs. 10(c)-10(d) vs. Figs. 10(e)-10(f)] and macro- [Figs. 11(a)-11(b) vs. Figs. 11(c)-11(d) and Figs. 12(a)-12(b) vs. Figs. 12(c)-12(d)] scale. Moreover, the plots of the far-field reflection patterns in Fig. 11 point out, on the one hand, the challenging nature of the addressed Shaped Coverage SPSSs problems while, on the other hand, they prove the feasibility of SPSSs that focus selectively in narrow angular regions. The effectiveness of using a SPSS in a SEE context can be better appreciated when analyzing the footprint power densities (Fig. 12). With reference to the HFSS simulations of the full-scale layouts, the reflected power is properly directed towards the user-defined coverage regions, regardless of their "dual beam" [Fig. 12(a) and Fig. 12(c)] or "low grazing" [Fig. 12(b) and Fig. 12(d)] nature, with limited power losses outside $\Theta$ (Fig. 11). The quantitative assessment of such a behavior is given by the corresponding values of the efficiency index $\xi_{H F S S}^{s h a}\left(\xi^{s h a} \triangleq \frac{4 \pi}{\Theta} \frac{\int_{\Theta}|\mathbf{F}(\mathbf{r} ; \mathbf{G})|^{2} r^{2} \sin (\theta) \mathrm{d} \theta \mathrm{d} \varphi}{\int_{0}^{2 \pi} \int_{0}^{\pi}|\mathbf{F}(\mathbf{r} ; \mathbf{G})|^{2} r^{2} \sin (\theta) \mathrm{d} \theta \mathrm{d} \varphi}\right.$ being the average directivity in the footprint region for the SEE shaped coverage) being $\xi_{H F S S}^{\text {sha }(\text { Two-Squares })}=18.81$ [dB] [Fig. $12(c)]$ and $\xi_{H F S S}^{\text {sha }(\text { Street }+ \text { Square })}=20.92[\mathrm{~dB}][$ Fig. $12(d)]$.

The next example is aimed at answering to the following questions: "Widening the aperture, the patterned surface similarity observed in the pencil coverage is still maintained when dealing with complex shaped footprints?" and "Does the design rule $\delta \Xi \approx \delta \xi$ apply also for complex shaped coverages?". Towards this end, a $P \times Q=75 \times 75$ square lattice comprising $\approx 5600$ UCs with an extension of $\Xi \approx 3.2 \times 3.2\left[\mathrm{~m}^{2}\right]$ [Fig. 13(a)] has been chosen to radiate the "StreetSquare" footprint and the performance of the arising layout have been compared with those of the smaller (i.e., $P \times Q=50 \times 50$ ) arrangement in Fig. 10(b). By taking a look to the sketch of the SPSS in Fig. 13(a), the answer to the first question is that, unlike the Pencil Coverage case, the increase of the size seems to imply a "stretching" of the $U C$ s distribution [Fig. 13(a) vs. Fig. $10(b)$ ] rather than a "repetition" of the same spatial behavior [Fig. 8(a) vs. Fig. 5(a)] probably because of the need of affording more complex wave manipulation phenomena to generate the complex footprint at hand. As for the second question, let us compare the radiation behavior of the two SPSSs in Fig. 10(b) and Fig. 13(a) pictorially described by the footprint color-maps in Fig. 12(d) and Fig. 13(b), respectively. Both layouts fulfil the requirement of reflecting the incident power towards the desired region $\Theta$. This happens more and more as the SPSS support enlarges according to the rule-of-thumb $\delta \xi^{s h a} \approx \delta \Xi$. Indeed, $\delta \Xi \approx 3.5[\mathrm{~dB}]$ and $\delta \xi_{H F S S}^{\text {sha }} \approx 3.2$ 
$[\mathrm{dB}]$ (i.e., $\left.\xi_{H F S S}^{s h a}\right\rfloor_{P=Q=75}=23.94[\mathrm{~dB}]\left[\text { Fig. 13(b)] and } \xi_{H F S S}^{s h a}\right\rfloor_{P=Q=50}=20.92[\mathrm{~dB}][$ Fig. $12(d)])$.

Whether the feasibility and the effectiveness of a SPSS deployment are key items to be addressed towards the implementation of a $S E E$, certainly the sustainable installation in a living environment (e.g., a town-square of a city or a room in a building floor) is a relevant issue, as well. Without pretending to give "the" solution to this problem, but just for adding some insights on the topic, some tests in the underlying numerical analysis have been devoted to this line of reasoning. More specifically, a representative example dealt with the possibility to realize, on a standard "Junior Poster Billboard" support [i.e., $\Xi \approx 3.6 \times 1.8\left[\mathrm{~m}^{2}\right]$ - Fig. 14(a)], a SPSS focusing the beam on the same "Street-Square" region $\Theta$ of the previous examples. Subject to the size constraint, the SPSS has been designed by optimizing the descriptors of $P \times Q=84 \times 42$ UCs. Figure 14 shows the synthesized layout [Fig. 14(a)] along with the HFSS-simulated footprint pattern [Fig. 14(b)]. As hoped, the radiated beam fulfils the mask requirements with a good accuracy being $\left.\xi_{H F S S}^{s h a}\right\rfloor_{P \times Q=84 \times 42}=21.30$ [dB] [Fig. 14(b)], even though, unlike Fig. 13(b), some spill-over effects appear at low grazing angles (i.e., $y_{\text {glob }}>140$ [m]) [Fig. 14(b)] due to the smaller vertical size of the SPSS [Fig. 14(a) vs. Fig. 13(a)].

The last test case is not concerned with a realistic SEE problem, but it is more aimed at giving the flavour of what it can be done with just a static and passive structure provided you have at disposal a suitable tool for managing the huge computational complexity of a high-dimension optimization problem. Accordingly, the proposed SPSS design has been applied to optimize a $P \times Q=75 \times 75 U C$ s arrangement, located $H=20[\mathrm{~m}]$ on the floor, for beaming a "Olympic Flag"-shaped region of extension $-150[\mathrm{~m}] \leq x_{\text {glob }} \leq 70[\mathrm{~m}]$ and $50[\mathrm{~m}] \leq y_{\text {glob }} \leq 150[\mathrm{~m}]$ (Fig. 1). The plots of the synthesized layout [Fig. 15(a)] and of the HFSS-computed distribution of the power reflected on the ground [Fig. 15(b)] prove the feasibility of a SPSS matching hard pattern-mask requirements $\left[\xi_{H F S S}^{s h a} \approx 18.3[\mathrm{~dB}]\right.$ - Fig. $\left.15(b)\right]$ as well as the capability of the proposed design method to efficiently deal with large-scale optimization problems. 


\section{Conclusions}

The feasibility of SPSSs with advanced wave manipulation properties has been addressed. A two-step design process, which combines the solution of an $I S$ problem to determine the surface currents affording user-desired pattern-mask constraints together with a $S b D$-based optimization of the corresponding patterned layout, has been proposed. Towards this end, an innovative scheme for building an $A I$-based $D T$ of the $U C$ s of the SPSS for predicting the EM response of this latter has been introduced. The arising synthesis method has been validated by considering different apertures, radiation objectives, and various operative SEE scenarios. Full-wave finite element simulations [24] of the synthesized layouts have been performed to assess the reliability of the synthesis results.

The outcomes from the numerical validation have demonstrated that the proposed $A I$-driven method allows the designer to reliably synthesize large SPSSs with excellent beam control capabilities by efficiently solving high-dimension optimization problems. The exploitation of a Local UC-DT within the SbD-based optimization of the SPSS layout assures a faithful prediction of the EM behavior of the non-uniform modulated patterned surface, while minimizing the computational costs of the solution of the forward problem at hand. The synthesis layouts fulfil challenging radiation objectives despite the choice of a basic $U C$ with a limited phase control. Future works, beyond the scope of the present paper, will be aimed at exploring the potentialities of the proposed method when using more complex $U C$ s (e.g., a higher number of per-element $D o F$ s or multi-layer structures) and tiled architectures instead of single-panel layouts. Moreover, the extension of the proposed method to real-time reconfigurable smart skins is currently under development.

\section{Acknowledgements}

This work benefited from the networking activities carried out within the Project "Cloaking Metasurfaces for a New Generation of Intelligent Antenna Systems (MANTLES)" (Grant No. 2017BHFZKH) funded by the Italian Ministry of Education, University, and Research under the PRIN2017 Program (CUP: E64I19000560001). Moreover, it benefited from the networking 
activities carried out within the Project "SPEED" (Grant No. 61721001) funded by National Science Foundation of China under the Chang-Jiang Visiting Professorship Program, the Project 'Inversion Design Method of Structural Factors of Conformal Load-bearing Antenna Structure based on Desired EM Performance Interval' (Grant no. 2017HZJXSZ) funded by the National Natural Science Foundation of China, and the Project 'Research on Uncertainty Factors and Propagation Mechanism of Conformal Loab-bearing Antenna Structure' (Grant No. 2021JZD003) funded by the Department of Science and Technology of Shaanxi Province within the Program Natural Science Basic Research Plan in Shaanxi Province. A. Massa wishes to thank E. Vico for her never-ending inspiration, support, guidance, and help.

\section{Appendix}

\section{Small-Scale/Full-Scale Mapping Rules}

The mapping rules between the predictions of the local surface susceptibility tensors of the small-scale $\operatorname{SPSS},\left\{\overline{\bar{\zeta}}_{p^{\prime} q^{\prime}}^{e / m}\left(\mathbf{G}^{\prime}\right) ; p^{\prime}=1, \ldots, P^{\prime} ; q^{\prime}=1, \ldots, Q^{\prime}\right\}$, and the predictions of the local surface susceptibility tensors of the full-scale $\operatorname{SPSS},\left\{\overline{\bar{\zeta}}_{p q}^{e / m}(\mathbf{G}) ; p=1, \ldots, P ; q=1, \ldots, Q\right\}$, which estimate the corresponding actual values, $\left\{\overline{\bar{\psi}}_{p q}^{e / m}(\mathbf{G}) ; p=1, \ldots, P ; q=1, \ldots, Q\right\}$, are defined as follows

$$
\overline{\bar{\zeta}}_{p q}^{e / m}(\mathbf{G})= \begin{cases}\left.\overline{\bar{\zeta}}_{p^{\prime} q^{\prime}}^{e / m}\left(\mathbf{G}^{\prime}\right)\right]_{p_{p^{\prime}=p} q^{\prime}=q} & \text { if }(p, q) \in \mathcal{C}^{(0)} \\ \left.\overline{\bar{\zeta}}_{p^{\prime} q^{\prime}}^{e / m}\left(\mathbf{G}^{\prime}\right)\right]_{p^{\prime}=p-P+P^{\prime}}^{q^{\prime}=q-Q+Q^{\prime}} & \text { if }(p, q) \in \mathcal{C}^{(1)} \\ \left.\overline{\bar{\zeta}}_{p^{\prime} q^{\prime}}^{e / m}\left(\mathbf{G}^{\prime}\right)\right]_{p^{\prime}=p}^{q^{\prime}=q-Q+Q^{\prime}} & \text { if }(p, q) \in \mathcal{C}^{(2)} \\ \left.\overline{\bar{\zeta}}_{p^{\prime} q^{\prime}}^{e / m}\left(\mathbf{G}^{\prime}\right)\right]_{p_{p^{\prime}=p-P+P^{\prime}}^{q^{\prime}=q}} & \text { if }(p, q) \in \mathcal{C}^{(3)} \\ \left.\bar{\zeta}_{p^{\prime} q^{\prime}}^{e / m}\left(\mathbf{G}^{\prime}\right)\right]_{p^{\prime}=p}^{q^{\prime}=3} & \text { if }(p, q) \in \mathcal{C}^{(4)} \\ \left.\bar{\zeta}_{p^{\prime} q^{\prime}}^{e / m}\left(\mathbf{G}^{\prime}\right)\right]_{p^{\prime}=3}^{q^{\prime}=q} & \text { if }(p, q) \in \mathcal{C}^{(5)} \\ \left.\overline{\bar{\zeta}}_{p^{\prime} q^{\prime}}^{e / m}\left(\mathbf{G}^{\prime}\right)\right]_{p^{\prime}=3}^{q^{\prime}=3} & \text { if }(p, q) \in \mathcal{C}^{(6)}\end{cases}
$$


where $\mathcal{C}^{(0)}=\{(p, q): p \in[1,2], q \in[1,2]\}, \mathcal{C}^{(1)}=\{(p, q): p \in[P-1, P], q \in[Q-1, Q]\}$, $\mathcal{C}^{(2)}=\{(p, q): p \in[1,2], q \in[Q-1, Q]\}, \mathcal{C}^{(3)}=\{(p, q): p \in[P-1, P], q \in[1,2]\}, \mathcal{C}^{(4)}=$ $\{(p, q): p=\{1,2, P-1, P\}, q \in[3, Q-2]\}, \mathcal{C}^{(5)}=\{(p, q): p \in[3, P-2], q=\{1,2, Q-1, Q\}\}$, and $\mathcal{C}^{(6)}=\{(p, q): p \in[3, P-2], q \in[3, Q-2]\}$ (Fig. 2).

\section{References}

[1] E. Basar, M. Di Renzo, J. De Rosny, M. Debbah, M.-S. Alouini, and R. Zhang, "Wireless communications through reconfigurable intelligent surfaces," IEEE Access, vol. 7, pp. 116753-116773, 2019.

[2] M. Di Renzo, M. Debbah, D.-T. Phan-Huy, A. Zappone, M.-S. Alouini, C. Yuen, V. Sciancalepore, G. C. Alexandropoulos, J. Hoydis, H. Gacanin, J. De Rosny, A. Bounceur, G. Lerosey, and M. Fink, "Smart radio environments empowered by reconfigurable AI metasurfaces: An idea whose time has come," EURASIP J. Wireless Commun. Netw., vol. 129, pp. 1-20, 2019.

[3] M. Di Renzo, A. Zappone, M. Debbah, M.-S. Alouini, C. Yuen, J. De Rosny, and S. Tretyakov, "Smart radio environments empowered by reconfigurable intelligent surfaces: How it works, state of research, and the road ahead," IEEE J. Sel. Areas Comm., vol. 38, no. 11 , pp. 2450-2525, Nov. 2020.

[4] A. Massa and M. Salucci, "On the design of complex EM devices and systems through the system-by-design paradigm - A framework for dealing with the computational complexity," IEEE Trans. Antennas Propag. (in press, 2021). Available: https://arxiv.org/abs/2107.09521.

[5] M. Di Renzo, K. Ntontin, J. Song, F. H. Danufane, X. Qian, F. Lazarakis, J. De Rosny, D.-T. Phan-Huy, O. Simeone, R. Zhang, M. Debbah, G. Lerosey, M. Fink, S. Tretyakov, and S. Shamai, "Reconfigurable intelligent surfaces vs. relaying: Differences, similarities, and performance comparison," IEEE Open J. Comm. Soc., vol. 1, pp. 798-807, 2020. 
[6] W. Tang, J. Dai, M. Chen, X. Li, Q. Cheng, S. Jin, K. Wong, and T. J. Cui, "Subject editor spotlight on programmable metasurfaces: The future of wireless," IET Electron. Lett., vol. 55, no. 7, pp. 360-361, 2019.

[7] A. Massa, A. Benoni, P. Da Ru, S. K. Goudos, B. Li, G. Oliveri, A. Polo, P. Rocca, and M. Salucci, "Designing smart electromagnetic environments for next-generation wireless communications," Telecom, vol. 2, no. 2, pp. 213-221, 2021.

[8] A. Pitilakis, O. Tsilipakos, F. Liu, K. M. Kossifos, A. C. Tasolamprou, D.-H. Kwon, M. S. Mirmoosa, D. Manessis, N. V. Kantartzis, C. Liaskos, M. A. Antoniades, J. Georgiou, C. M. Soukoulis, M. Kafesaki, and S. A. Tretyakov, "A multi-functional reconfigurable metasurface: Electromagnetic design accounting for fabrication aspects," IEEE Trans. Antennas Propag., vol. 69, no. 3, pp. 1440-1454, Mar. 2021.

[9] C. Liaskos, S. Nie, A. Tsioliaridou, A. Pitsillides, S. Ioannidis, and I. Akyildiz, "A new wireless communication paradigm through software-controlled metasurfaces," IEEE Commun. Mag., vol. 56, no. 9, pp. 162-169, 2018.

[10] C. Huang, A. Zappone, G. C. Alexandropoulos, M. Debbah, and C. Yuen, "Reconfigurable intelligent surfaces for energy efficiency in wireless communication," IEEE Trans. Wireless Commun., vol. 18, no. 8, pp. 4157-4170, 2019.

[11] G. Oliveri, P. Rocca, M. Salucci, and A. Massa, "Holographic smart EM skins for advanced beam power shaping in next generation wireless environments," IEEE J. Multiscale Multiphysics Computat. Techn. (under review, 2021). Available: https://arxiv.org/abs/2106.10932.

[12] F. Yang and Y. Rahmat-Samii, Surface Electromagnetics with Applications in Antenna, Microwave, and Optical Engineering, Cambridge, UK: Cambridge University Press, 2019.

[13] G. Oliveri, D. H. Werner, and A. Massa, "Reconfigurable electromagnetics through metamaterials - A review," Proc. IEEE, vol. 103, no. 7, pp. 1034-1056, Jul. 2015. 
[14] G. Oliveri, F. Viani, N. Anselmi, and A. Massa, "Synthesis of multi-layer WAIM coatings for planar phased arrays within the system-by-design framework," IEEE Trans. Antennas Propag., vol. 63, no. 6, pp. 2482-2496, Jun. 2015.

[15] G. Oliveri, A. Gelmini, A. Polo, N. Anselmi, and A. Massa, "System-by-design multiscale synthesis of task-oriented reflectarrays," IEEE Trans. Antennas Propag., vol. 68, no. 4, pp. 2867-2882, Apr. 2020.

[16] A. Massa, G. Oliveri, P. Rocca, and F. Viani, "System-by-Design: a new paradigm for handling design complexity," Proc. 8th European Conf. Antennas Propag., The Hague, The Netherlands, 2014, pp. 1180-1183.

[17] M. A. Ricoy and J. L. Volakis, "Derivation of generalized transition/boundary conditions for planar multiple-layer structures," Radio Sci., vol. 25, pp. 391-405, 1990.

[18] E. F. Kuester, M. A. Mohamed, and C. L. Holloway, "Averaged transition conditions for electromagnetic fields at a metafilm," IEEE Trans. Antennas Propag., vol. 51, no. 10, pt. 1, pp. 2641-2651, Oct. 2003.

[19] K. Achouri, M. A. Salem, and C. Caloz, "General metasurface synthesis based on susceptibility tensors," IEEE Trans. Antennas Propag, vol. 63, no. 7, pp. 2977-2991, July 2015.

[20] P. Rocca, R. L. Haupt, and A. Massa, "Sidelobe reduction through element phase control in subarrayed array antennas," IEEE Antennas Wireless Propag. Lett., vol. 8, pp. 437-440, 2009.

[21] G. Oliveri, M. Salucci, N. Anselmi, and A. Massa, "Multiscale System-by-Design synthesis of printed WAIMs for waveguide array enhancement," IEEE J. Multiscale Multiphysics Computat. Techn., vol. 2, pp. 84-96, 2017.

[22] G. Oliveri, A. Polo, M. Salucci, G. Gottardi, and A. Massa, "SbD-based synthesis of lowprofile WAIM superstrates for printed patch arrays," IEEE Trans. Antennas Propag., vol. 69, no. 7, pp. 3849-3862, Jul. 2021. 
[23] M. Salucci, L. Tenuti, G. Oliveri, and A. Massa, "Efficient prediction of the EM response of reflectarray antenna elements by an advanced statistical learning method," IEEE Trans. Antennas Propag., vol. 66, no. 8, pp. 3995-4007, Aug. 2018.

[24] ANSYS Electromagnetics Suite - HFSS (2021). ANSYS, Inc.

[25] M. Salucci, A. Gelmini, G. Oliveri, N. Anselmi, and A. Massa, "Synthesis of shaped beam reflectarrays with constrained geometry by exploiting non-radiating surface currents," IEEE Trans. Antennas Propag., vol. 66, no. 11, pp. 5805-5817, Nov. 2018.

[26] A. Osipov and S. Tretyakov, Modern electromagnetic scattering theory with applications. John Wiley \& Sons, 2017.

[27] I. V. Lindell and A. Sihvola, Boundary Conditions in Electromagnetics. IEEE Press, 2019.

[28] J. Huang and J. A. Encinar, Reflectarray Antennas. Wiley, 2008.

[29] P. Rocca, M. Benedetti, M. Donelli, D. Franceschini, and A. Massa, "Evolutionary optimization as applied to inverse problems," Inverse Prob., vol. 25, pp. 1-41, Dec. 2009.

[30] M. Ciydem and E. A. Miran, "Dual-polarization wideband sub-6 GHz suspended patch antenna for 5G base station," IEEE Antennas Wireless Propag. Lett., vol. 19, no. 7, pp. 1142-1146, Jul. 2020. 


\section{FIGURE CAPTIONS}

- Figure 1. Problem geometry. Sketch of the smart EM environment (SEE) scenario.

- Figure 2. Illustrative Example - Example of Local UC-DT mapping between the smallscale model $\left(P^{\prime} \times Q^{\prime}=5 \times 5\right)$ and the full-scale SPSS arrangement $(P \times Q=10 \times 10)$.

- Figure 3. Illustrative Example - Model of (a) the unit cell (UC) of the SPSS and of $(b)$ the small-scale SPSS layout. Plot of (c) the phase, $\angle \widetilde{R}_{T E, T E}$, and of $(d)$ the magnitude, $\left|\widetilde{R}_{T E, T E}\right|$, of the average " $T E-T E$ ” reflection coefficient of the central element $\left[\left(p^{\prime}, q^{\prime}\right)=\right.$ $\left.\left(\frac{P^{\prime}+1}{2}, \frac{Q^{\prime}+1}{2}\right)\right]$ of the small-scale SPSS layout.

- Figure 4. Illustrative Example (Pencil Coverage, $P=Q=30, \theta^{T}=50$ [deg], $\varphi^{T}=-8$ [deg], $H=5[\mathrm{~m}])$ - Plot of $(a)$ the evolution of the pattern matching index, $\Gamma_{i}(i=$ $1, \ldots, I)$ and of the $(b)$ reference surface current distribution (x-component), $J_{x}^{\text {opt }}(\mathbf{r}) \triangleq$ $\mathbf{J}_{\text {opt }}^{\text {tot }}(\mathbf{r}) \cdot \widehat{\mathbf{x}}(\mathbf{r} \in \Xi)$, with $(c)$ the radiated far-field pattern in the $(u, v)$ plane, $\left|\mathbf{F}_{\text {opt }}(\mathbf{r})\right|(1)$.

- Figure 5. Illustrative Example (Pencil Coverage, $P=Q=30, \theta^{T}=50$ [deg], $\varphi^{T}=-8$ [deg], $H=5[\mathrm{~m}])$ - Plot of (a) the synthesized SPSS layout and of the corresponding $(b)$ surface current distribution ( $x$-component), $J_{x}^{N}(\mathbf{r}) \triangleq \mathbf{J}_{N}^{t o t}(\mathbf{r}) \cdot \widehat{\mathbf{x}}(\mathbf{r} \in \Xi)$, and $(c)$ radiated far-field pattern in the $(u, v)$ plane, $\left|\mathbf{F}_{N}(\mathbf{r})\right|\left(\mathbf{F}_{N}(\mathbf{r} ; \mathbf{G}) \triangleq \mathcal{L}\left[\mathbf{J}_{N}^{t o t}(\mathbf{r} ; \mathbf{G})\right]\right)$ (1).

- Figure 6. Numerical Validation (Pencil Coverage, $P=Q=30, \theta^{T}=50$ [deg], $\varphi^{T}=$ $-8[\mathrm{deg}], H=5[\mathrm{~m}])$ - Plot of the HFSS-simulated (a) surface current distribution ( $x$ component), $J_{x}^{H F S S}(\mathbf{r}) \triangleq \mathbf{J}_{H F S S}^{t o t}(\mathbf{r}) \cdot \widehat{\mathbf{x}}(\mathbf{r} \in \Xi)$, and $(c)$ radiated far-field pattern in the $(u, v)$ plane, $\left|\mathbf{F}_{H F S S}(\mathbf{r})\right|\left(\mathbf{F}_{H F S S}(\mathbf{r} ; \mathbf{G}) \triangleq \mathcal{L}\left[\mathbf{J}_{H F S S}^{t o t}(\mathbf{r} ; \mathbf{G})\right]\right)$ (1).

- Figure 7. Numerical Validation (Pencil Coverage, $P=Q=30, \theta^{T}=50[\mathrm{deg}], \varphi^{T}=$ -8 [deg], $H=5[\mathrm{~m}])$ - Plots of $(a)(b)$ the SPSS footprint pattern and of $(c)(d)$ a component of the surface averaged electric field (5), $\left\{\mathbf{E}_{p q}^{\text {ave }}(\mathbf{G}) ; p=1, \ldots, P ; q=1, \ldots, Q\right\}$, $(a)(c)$ analytically-computed or $(b)(d) H F S S$-simulated.

- Figure 8. Numerical Validation (Pencil Coverage, $P=Q=50, \theta^{T}=50[\mathrm{deg}], \varphi^{T}=$ $-8[\mathrm{deg}], H=5[\mathrm{~m}])$ - Plot of $(a)$ the synthesized SPSS layout and of the corresponding 
(b) far-field pattern in the $(u, v)$ plane, $\left|\mathbf{F}_{N}(\mathbf{r})\right|\left(\mathbf{F}_{N}(\mathbf{r} ; \mathbf{G}) \triangleq \mathcal{L}\left[\mathbf{J}_{N}^{\text {tot }}(\mathbf{r} ; \mathbf{G})\right]\right)(1),(c)$ SPSS footprint pattern, $(d)$ surface current distribution ( $x$-component), $J_{x}^{N}(\mathbf{r}) \triangleq \mathbf{J}_{N}^{t o t}(\mathbf{r})$. $\widehat{\mathbf{x}}(\mathbf{r} \in \Xi)$, and $(e) y$-component of the surface averaged electric field $(5),\left\{\mathbf{E}_{p q}^{\text {ave }}(\mathbf{G})\right.$; $p=1, \ldots, P ; q=1, \ldots, Q\}$.

- Figure 9. Numerical Validation (Pencil Coverage, $P=Q=50, \theta^{T}=50$ [deg], $\varphi^{T}=$ -8 [deg], $H=5[\mathrm{~m}])$ - Plot of the HFSS-simulated (a) far-field pattern in the $(u, v)$ plane, $\left|\mathbf{F}_{H F S S}(\mathbf{r})\right|\left(\mathbf{F}_{H F S S}(\mathbf{r} ; \mathbf{G}) \triangleq \mathcal{L}\left[\mathbf{J}_{H F S S}^{t o t}(\mathbf{r} ; \mathbf{G})\right]\right)(1),(b) S P S S$ footprint pattern, (c) surface current distribution $\left(x\right.$-component), $J_{x}^{H F S S}(\mathbf{r}) \triangleq \mathbf{J}_{H F S S}^{t o t}(\mathbf{r}) \cdot \widehat{\mathbf{x}}(\mathbf{r} \in \Xi)$, and (e) $y$-component of the surface averaged electric field (5), $\left\{\mathbf{E}_{p q}^{a v e}(\mathbf{G}) ; p=1, \ldots, P\right.$; $q=1, \ldots, Q\}$.

- Figure 10. Numerical Validation (Shaped Coverage, $P=Q=50, H=5[\mathrm{~m}]$ ) - Plots of $(a)(b)$ the synthesized SPSS layouts along with the corresponding $(c)(d)$ analyticallycomputed or $(e)(f) H F S S$-simulated surface current distributions ( $x$-component), $J_{x}^{H F S S}(\mathbf{r}) \triangleq$ $\mathbf{J}_{H F S S}^{t o t}(\mathbf{r}) \cdot \widehat{\mathbf{x}}(\mathbf{r} \in \Xi)$ for $(a)(c)(e)$ the "Two-Square" and $(b)(d)(f)$ the "Street-Square" footprints.

- Figure 11. Numerical Validation (Shaped Coverage, $P=Q=50, H=5[\mathrm{~m}]$ ) - Plots of $(a)(b)$ the analytically-computed and $(c)(d)$ the HFSS-simulated far-field patterns in the $(u, v)$ plane, $|\mathbf{F}(\mathbf{r})|\left(\mathbf{F}(\mathbf{r} ; \mathbf{G}) \triangleq \mathcal{L}\left[\mathbf{J}^{t o t}(\mathbf{r} ; \mathbf{G})\right]\right)$ (1) for $(a)(c)$ the "Two-Square" and $(b)(d)$ the "Street-Square" footprints.

- Figure 12. Numerical Validation (Shaped Coverage, $P=Q=50, H=5[\mathrm{~m}]$ ) - Plots of $(a)(b)$ the analytically-computed and $(c)(d)$ the HFSS-simulated SPSS footprint patterns in correspondence with $(a)(c)$ the "Two-Square" and $(b)(d)$ the "Street-Square" footprint targets.

- Figure 13. Numerical Validation (Shaped Coverage, "Street-Square" footprint, $P=Q=$ $75, H=5[\mathrm{~m}])$ - Plot of (a) the synthesized SPSS layout and of (b) the HFSS-simulated SPSS footprint pattern.

- Figure 14. Numerical Validation (Shaped Coverage, "Street-Square" footprint, $P \times Q=$ 
$84 \times 42, H=5[\mathrm{~m}])-$ Plot of $(a)$ the synthesized SPSS layout and of $(b)$ the HFSSsimulated SPSS footprint pattern.

- Figure 15. Numerical Validation (Shaped Coverage, "Olympic Flag” footprint, $P=Q=$ $75, H=20[\mathrm{~m}])$ - Plot of (a) the synthesized SPSS layout and of (b) the HFSS-simulated SPSS footprint pattern. 


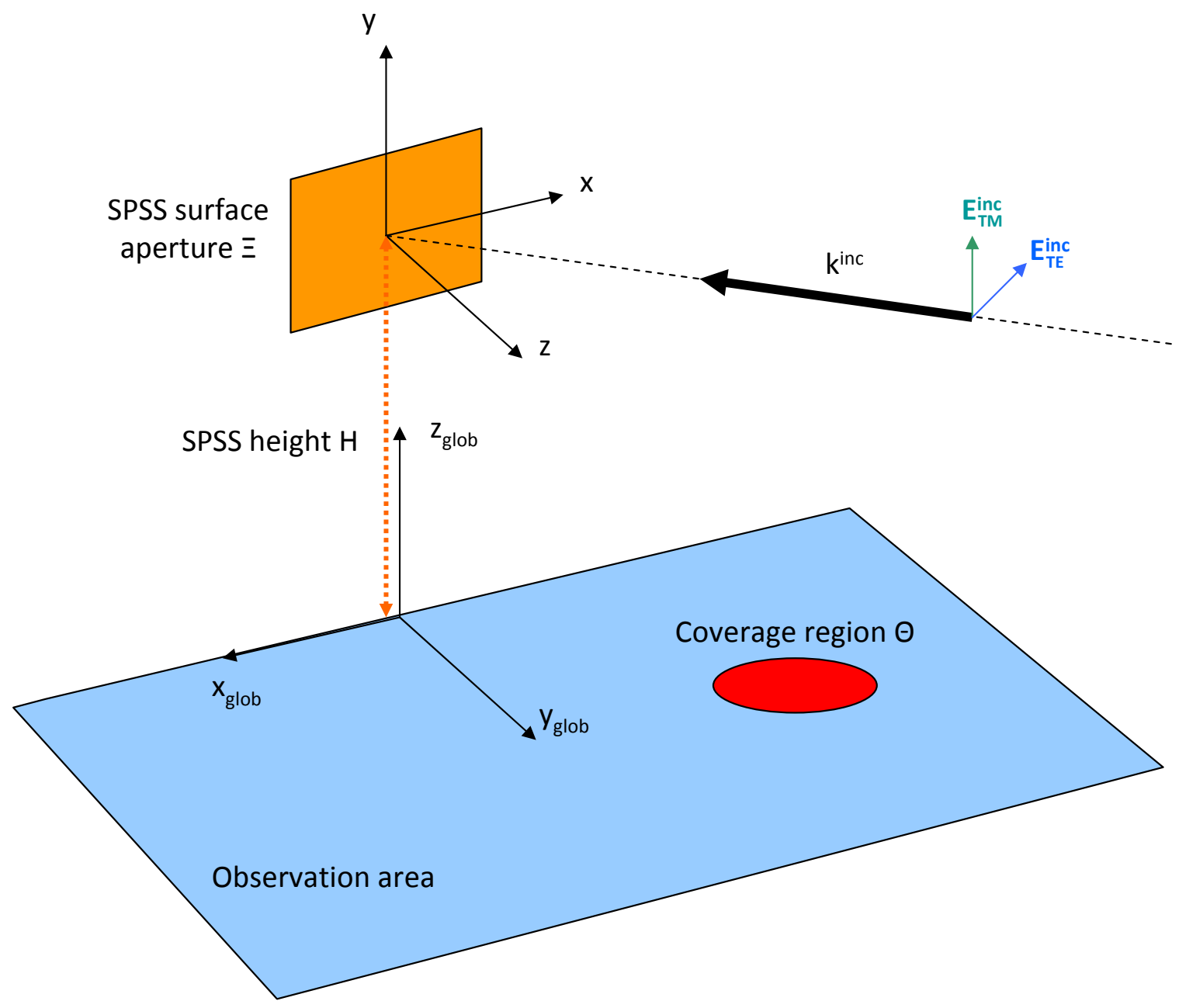

Fig. 1 - G. Oliveri et al., "Building a Smart EM Environment - AI-Enhanced Aperiodic ..." 


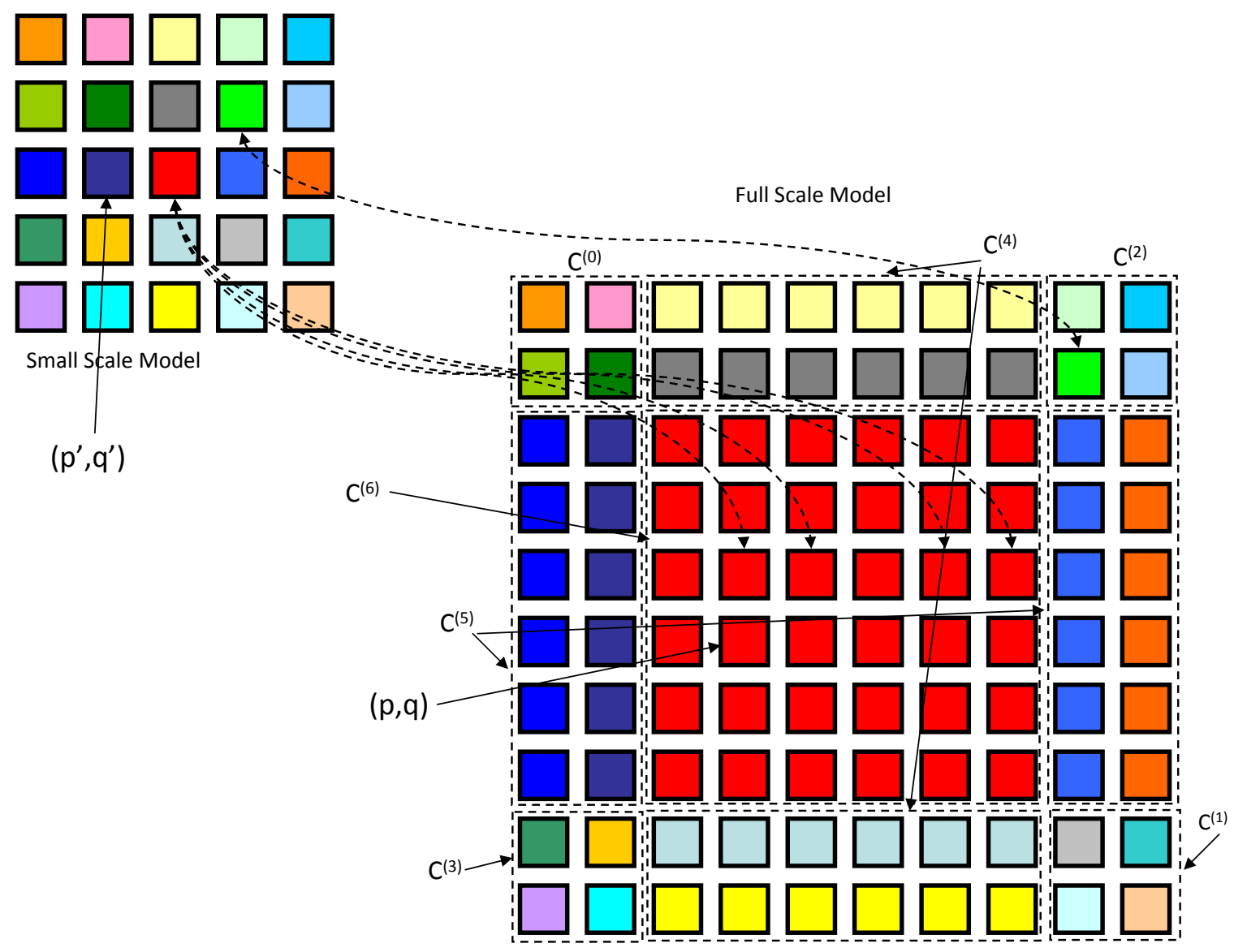

Fig. 2 - G. Oliveri et al., "Building a Smart EM Environment - AI-Enhanced Aperiodic ..." 


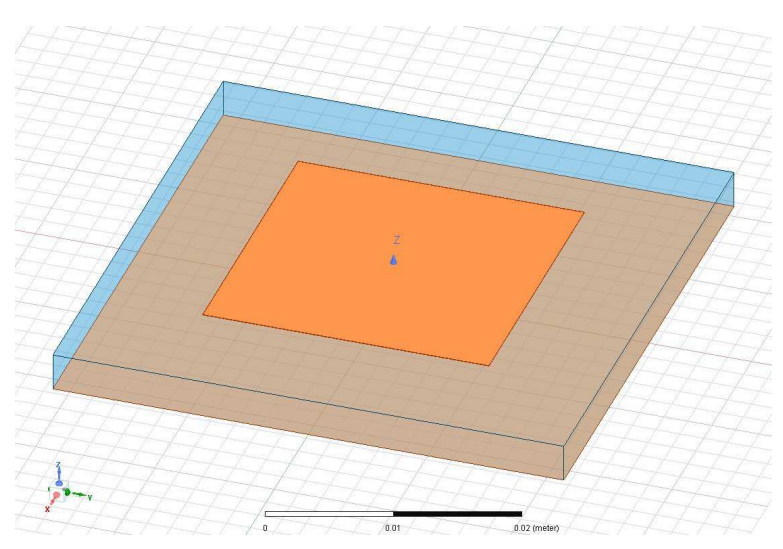

(a)

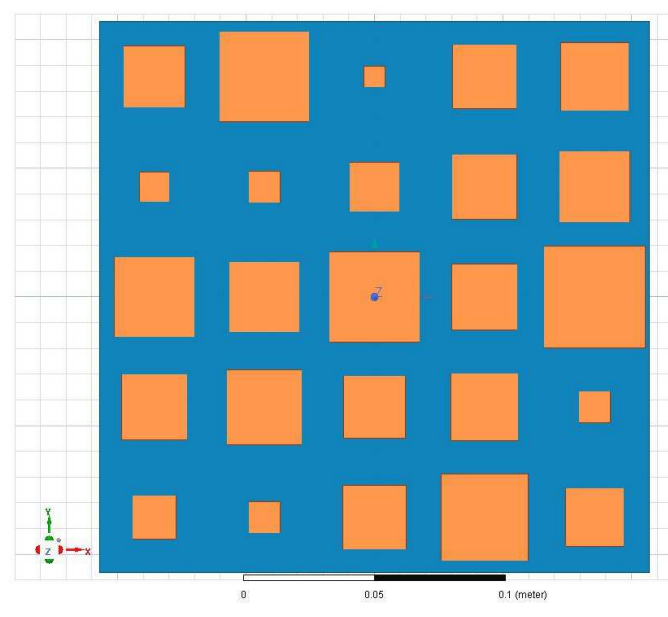

(b)

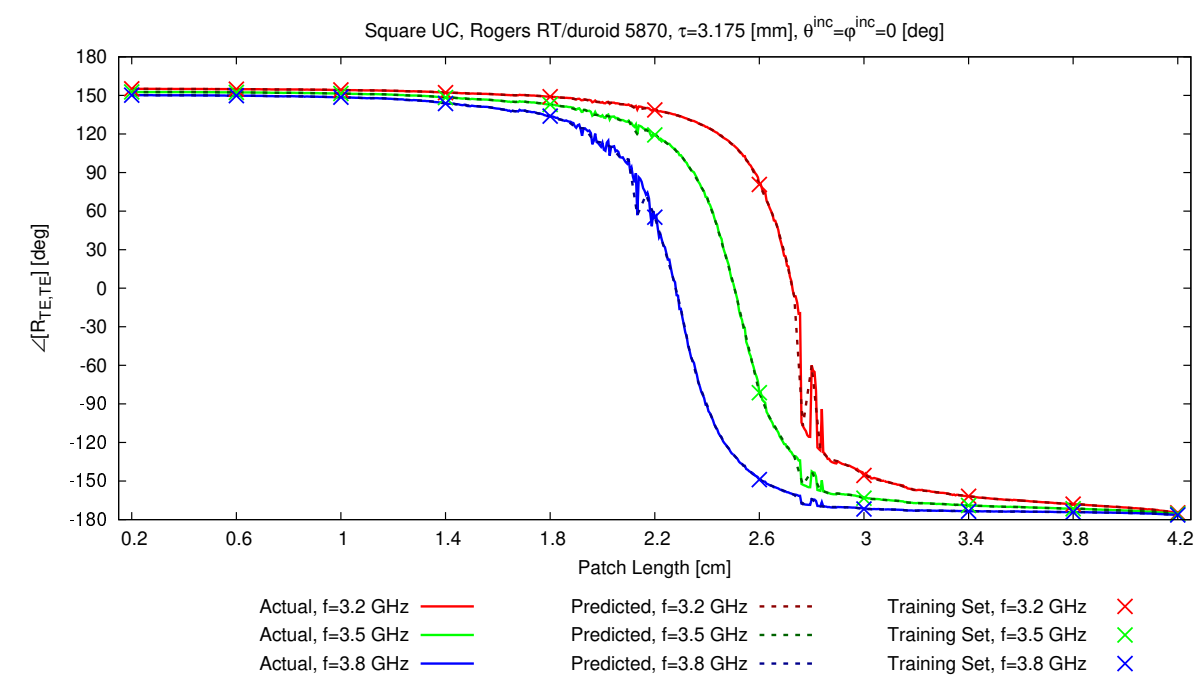

(c)

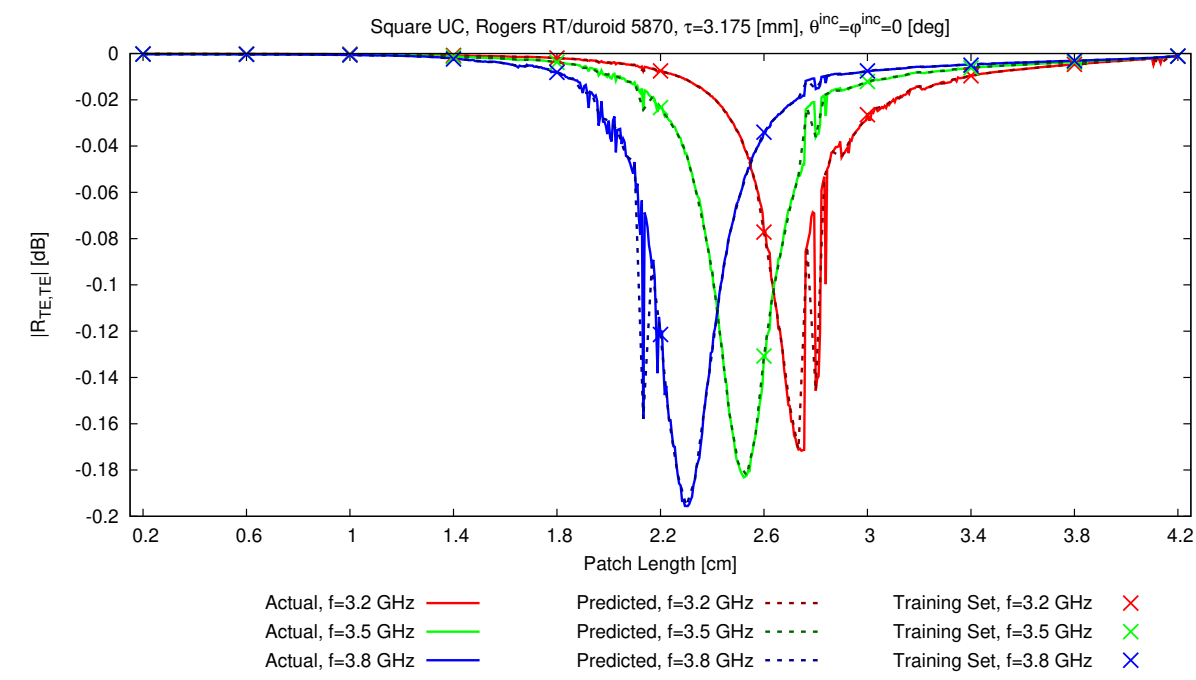

(d)

Fig. 3 - G. Oliveri et al., "Building a Smart EM Environment - AI-Enhanced Aperiodic ..." 


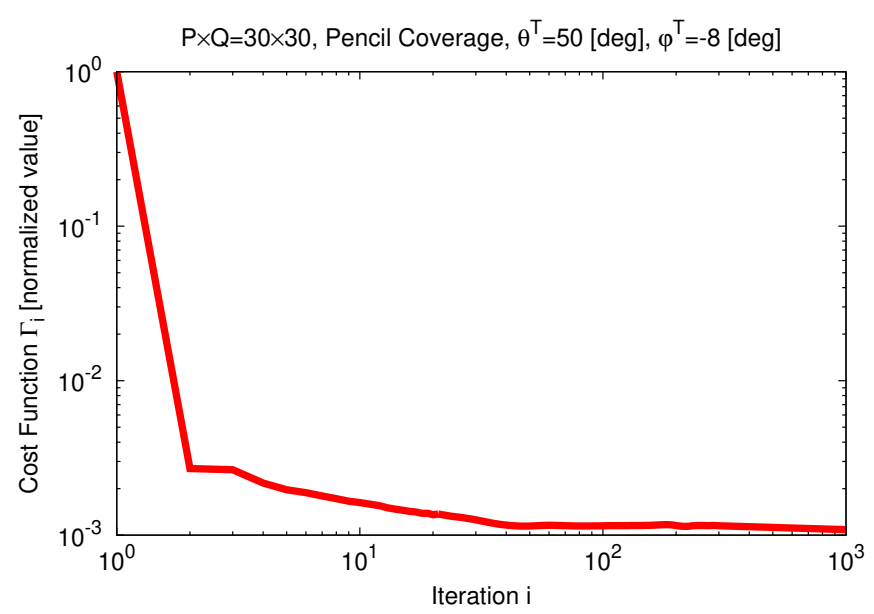

(a)

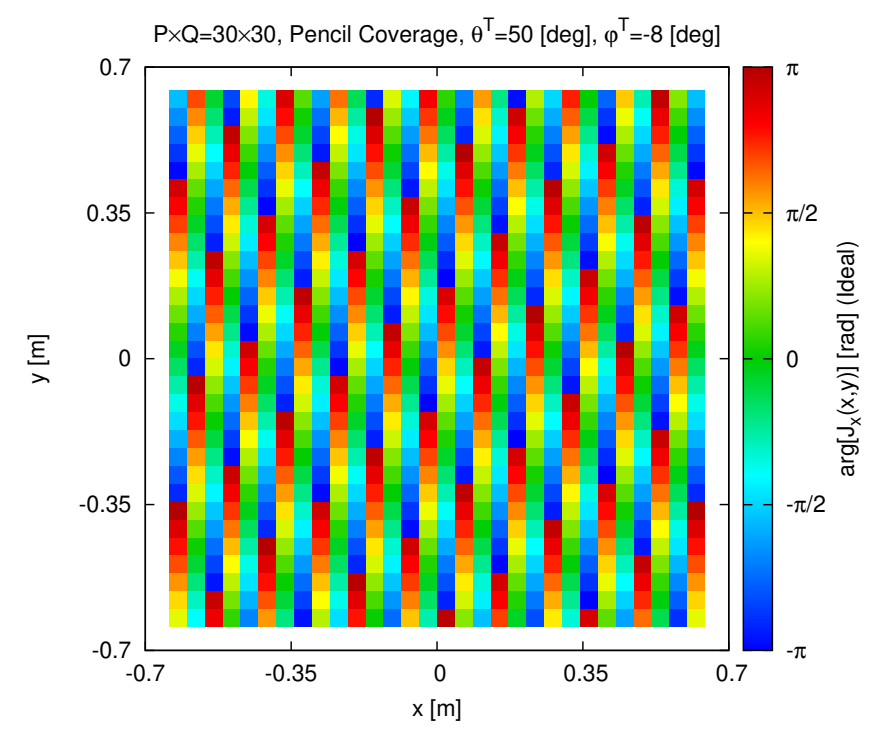

(b)

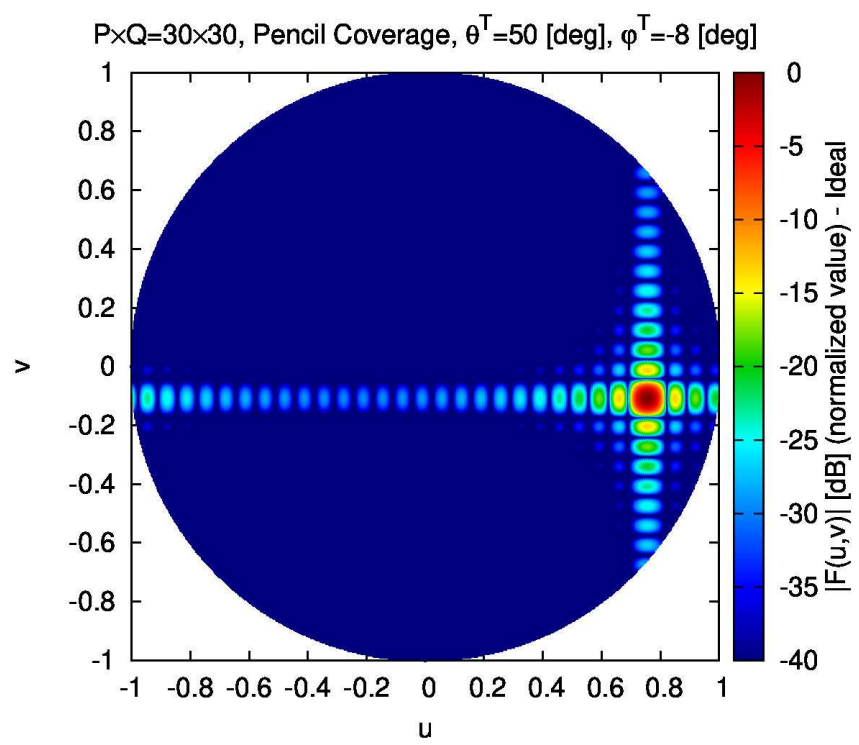

(c)

Fig. 4 - G. Oliveri et al., "Building a Smart EM Environment - AI-Enhanced Aperiodic ..." 


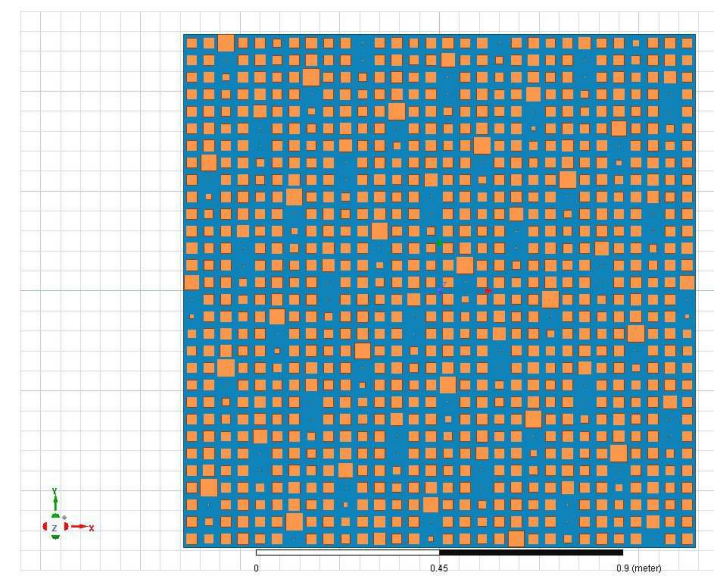

(a)

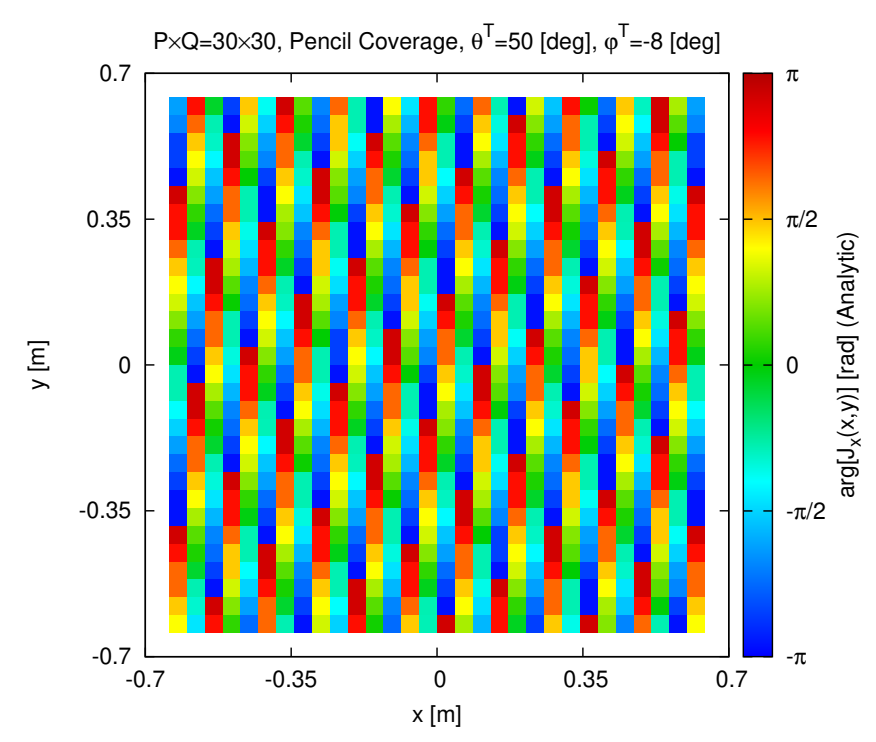

(b)

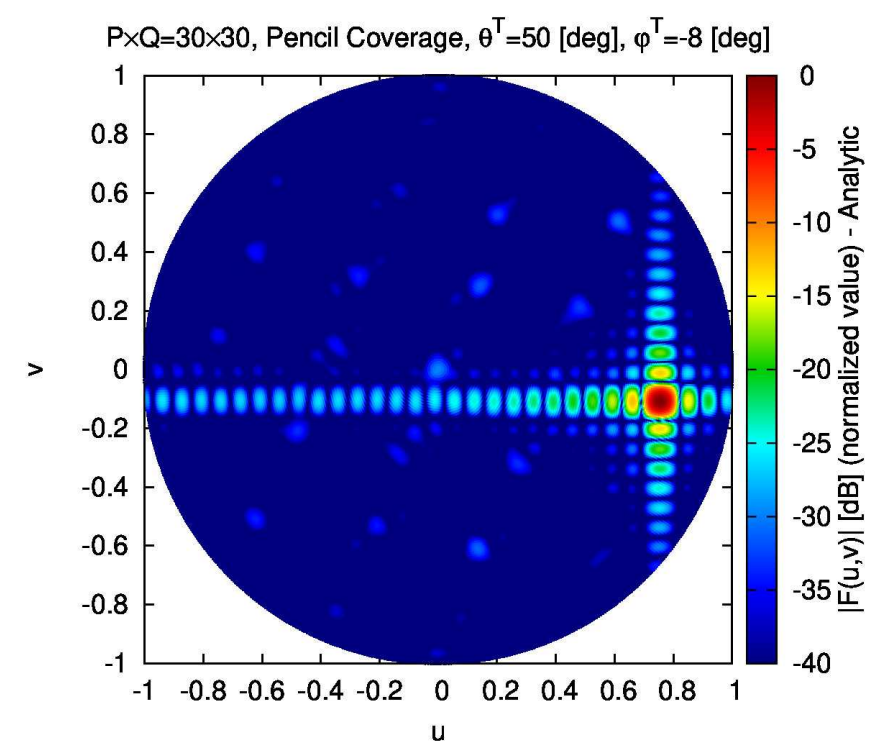

(c)

Fig. 5 - G. Oliveri et al., "Building a Smart EM Environment - AI-Enhanced Aperiodic ..." 


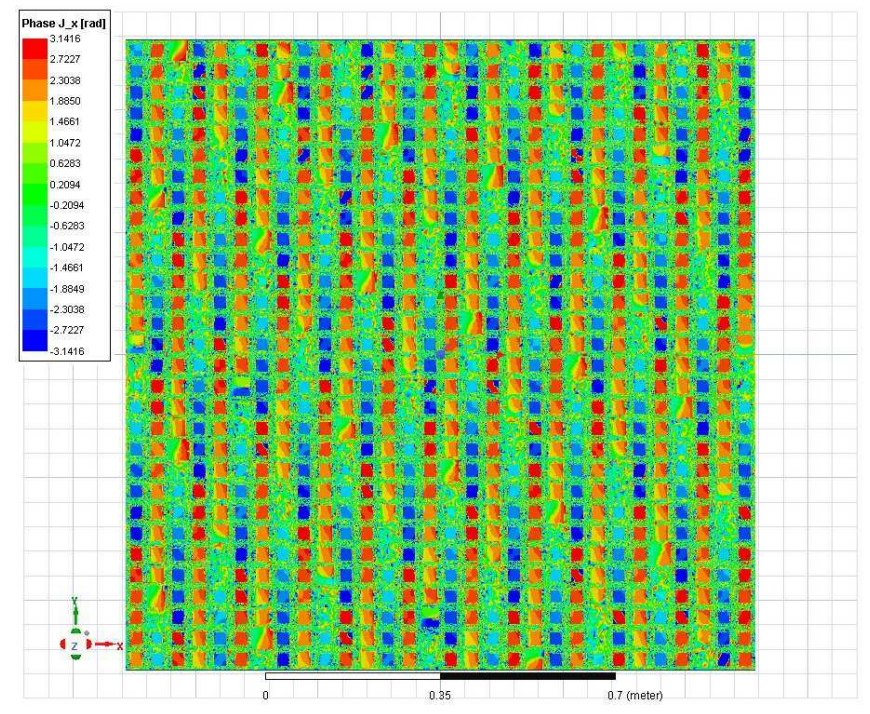

(a)

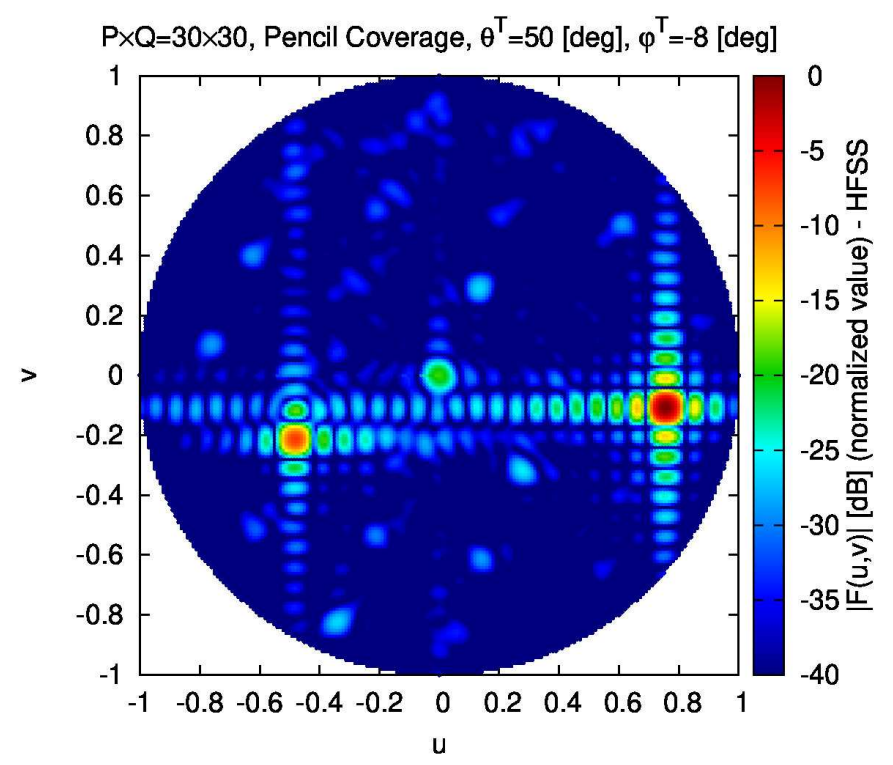

(b)

Fig. 6 - G. Oliveri et al., "Building a Smart EM Environment - AI-Enhanced Aperiodic ..." 


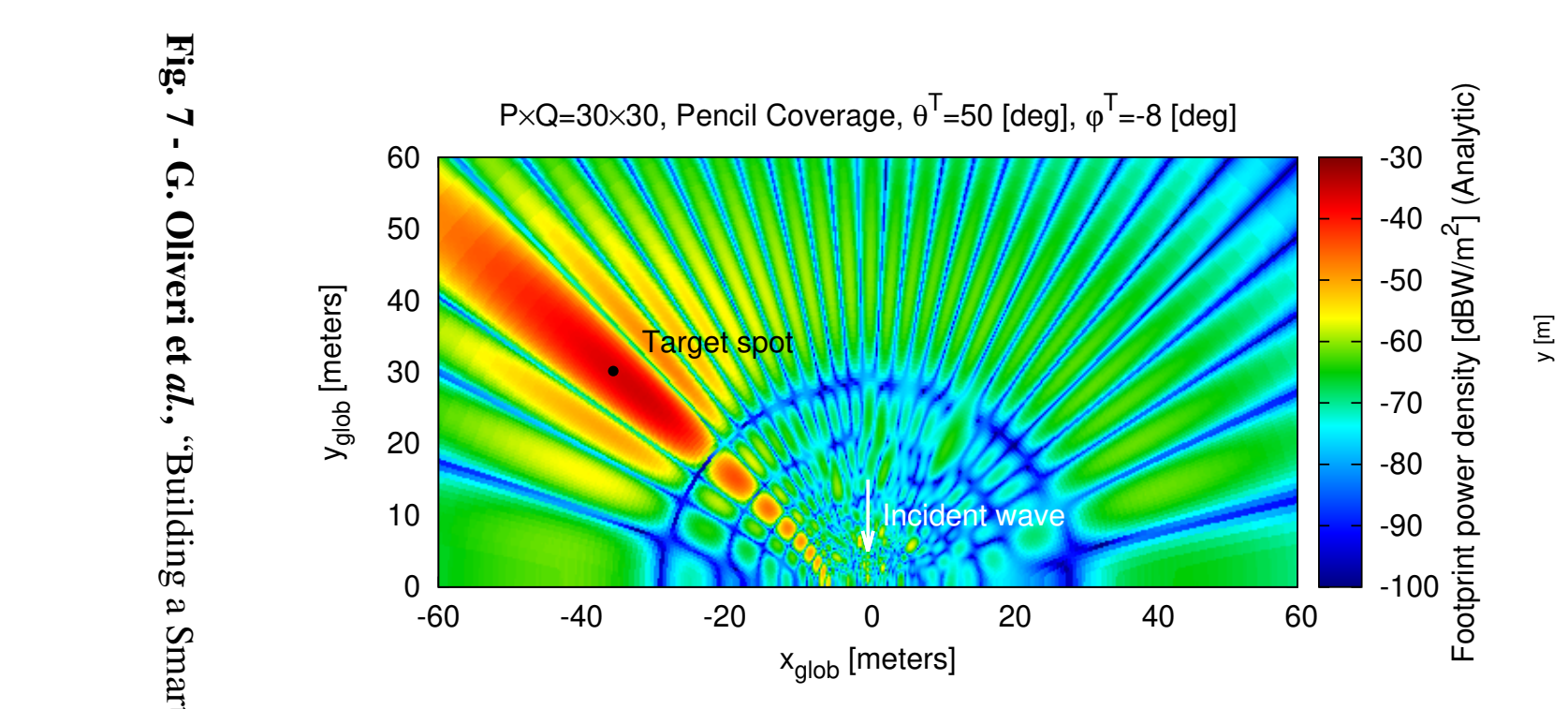

(a)

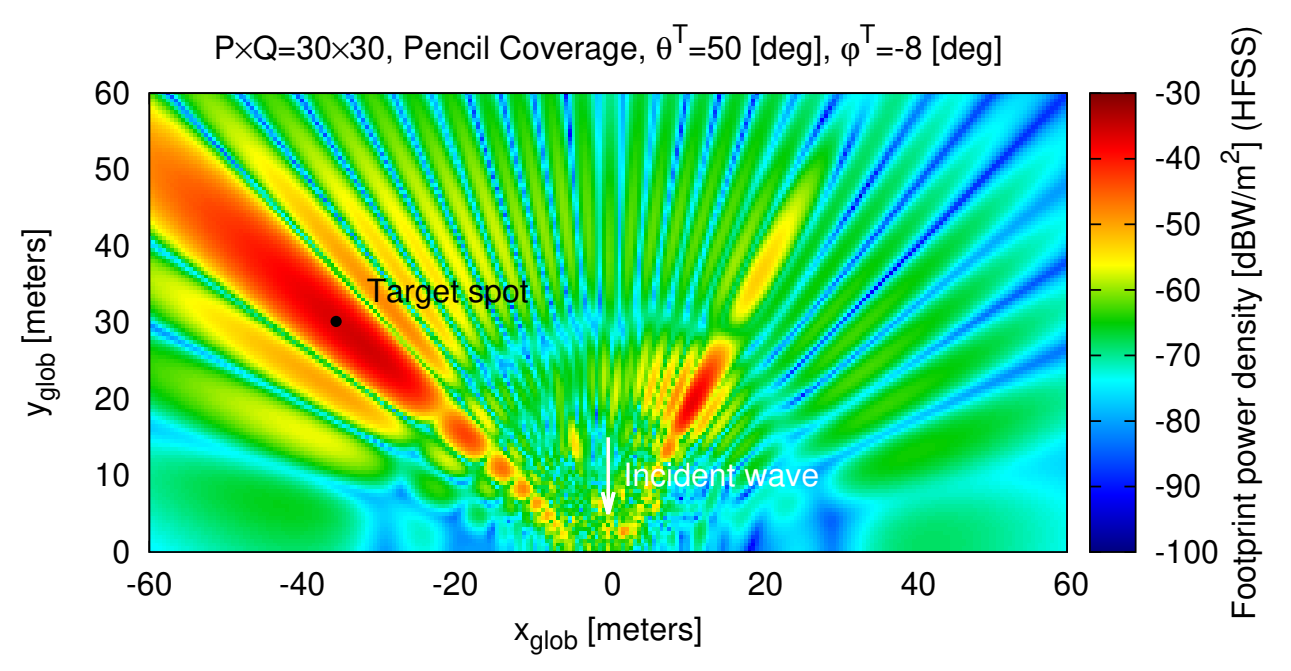

(b)

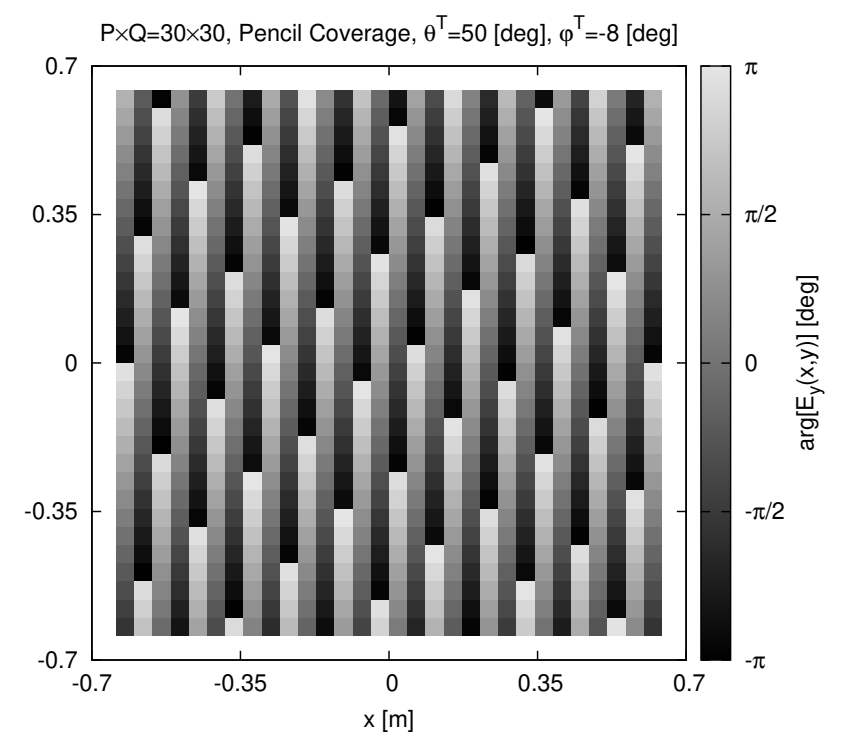

(c)

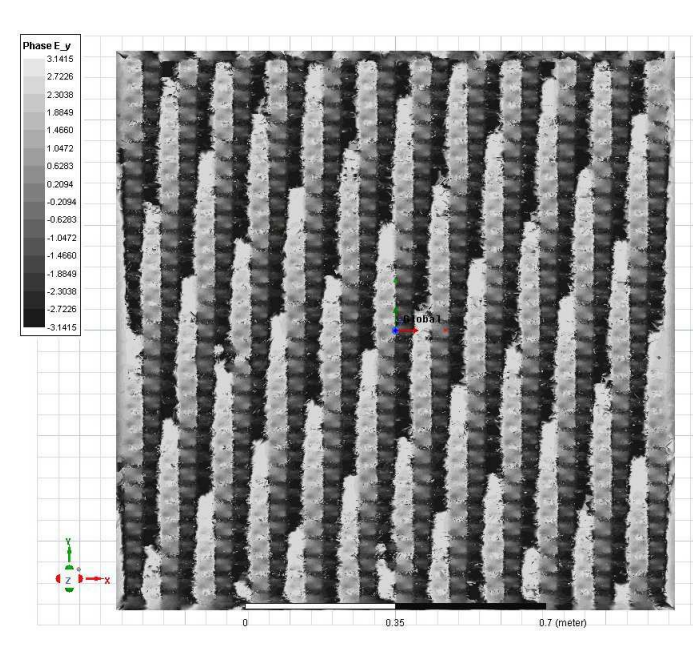

(d) 


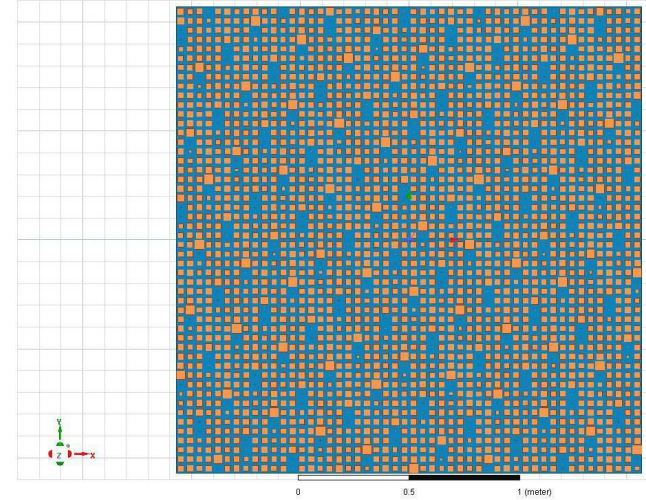

(a)

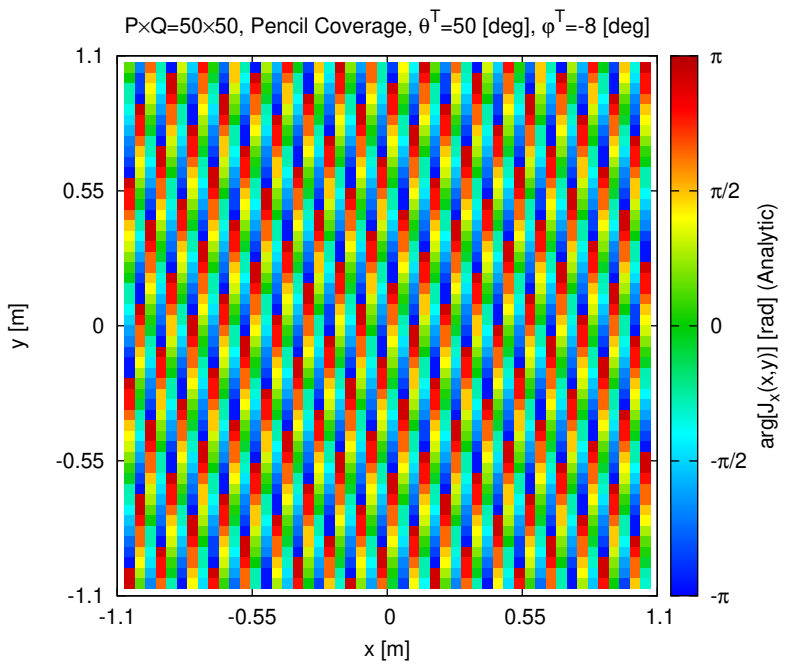

$(d)$

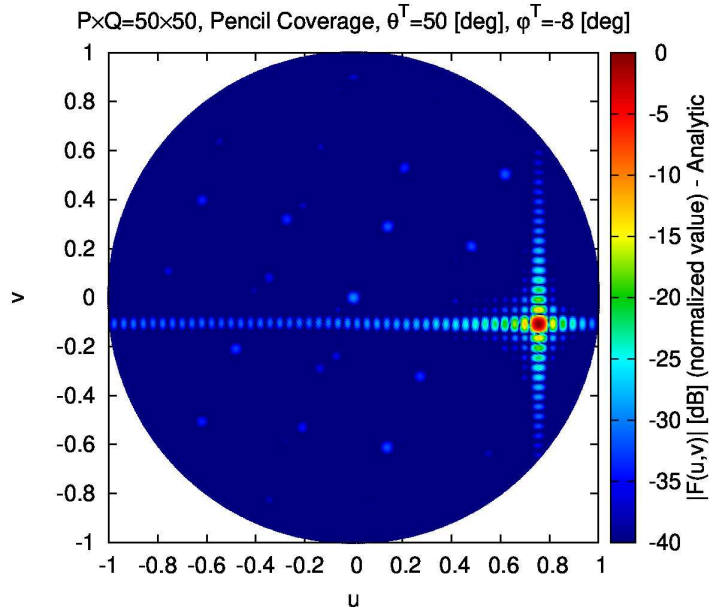

(b)

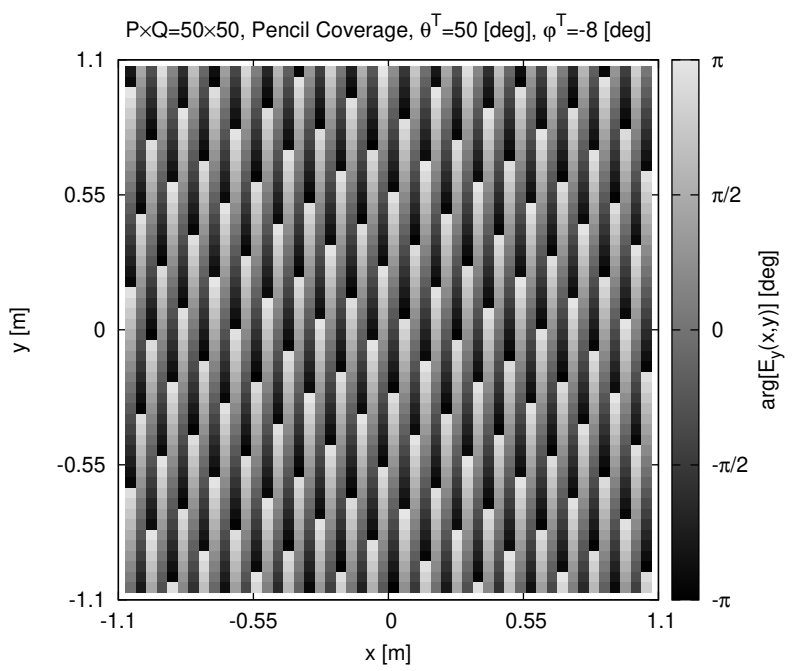

$(e)$

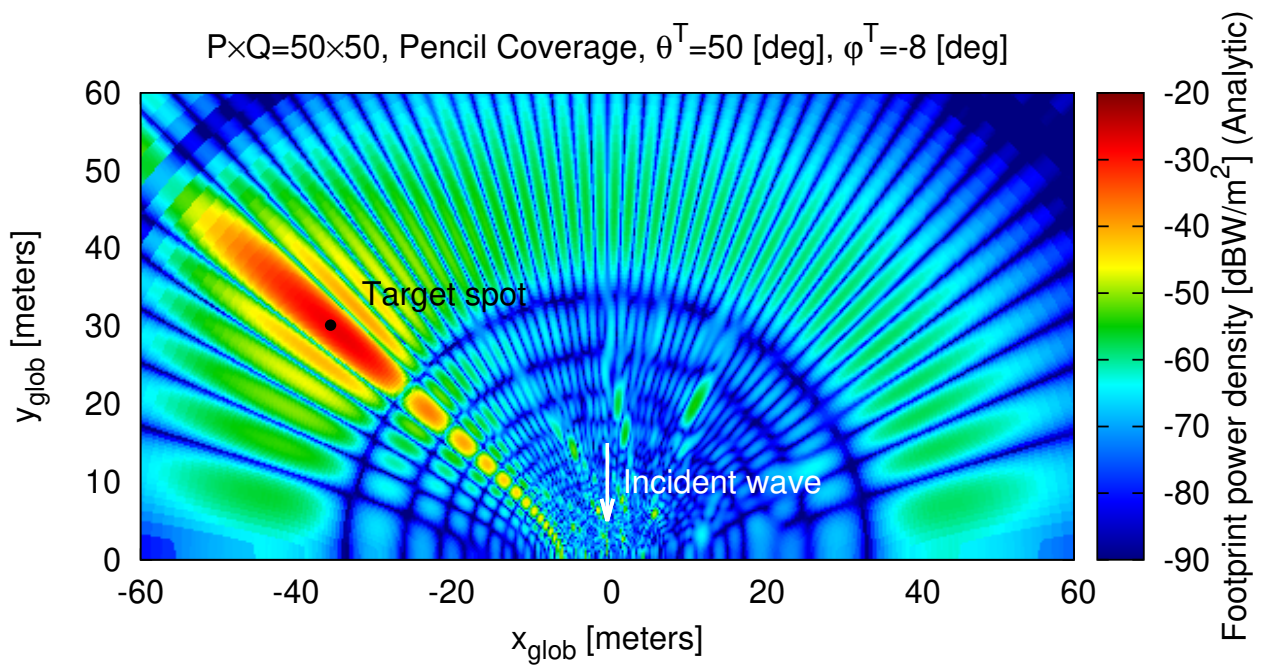

(c)

Fig. 8 - G. Oliveri et al., "Building a Smart EM Environment - AI-Enhanced Aperiodic ..." 


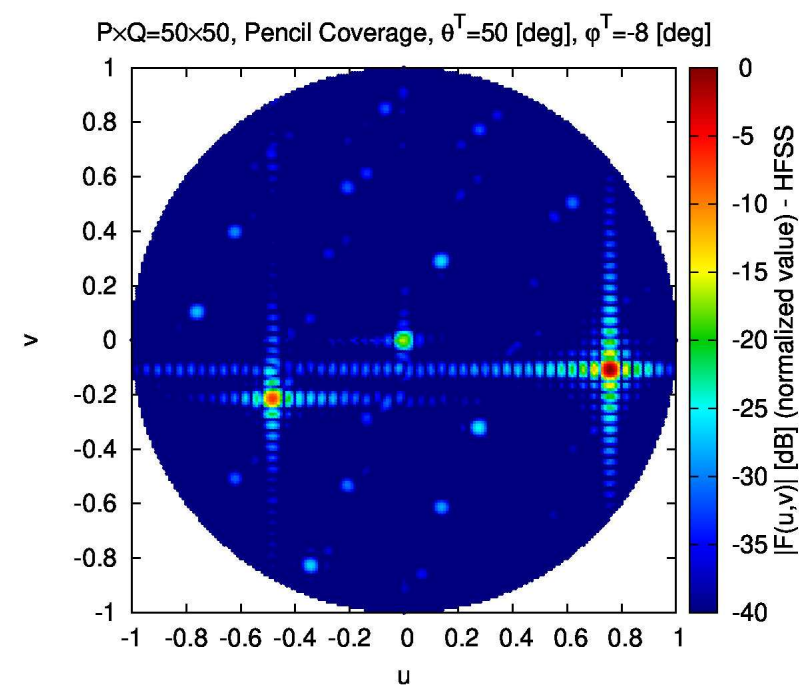

(a)

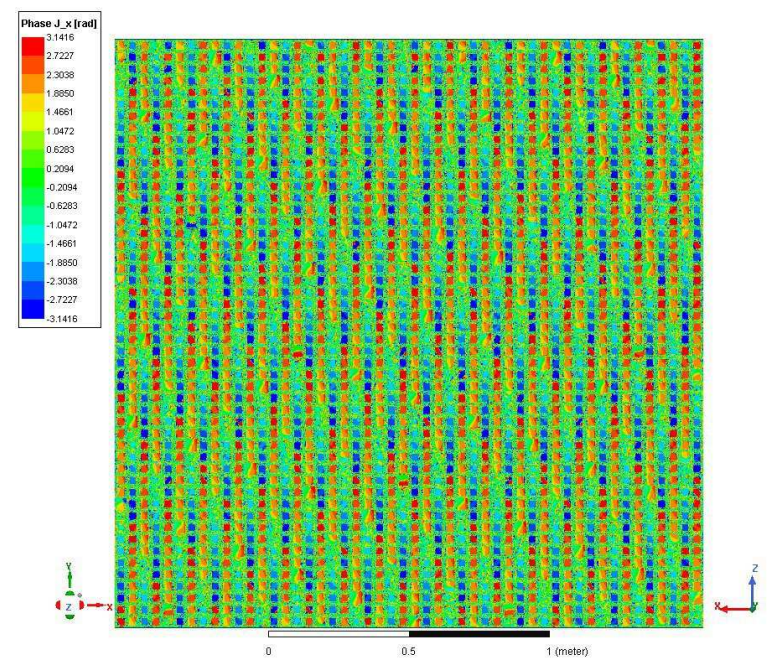

(c)

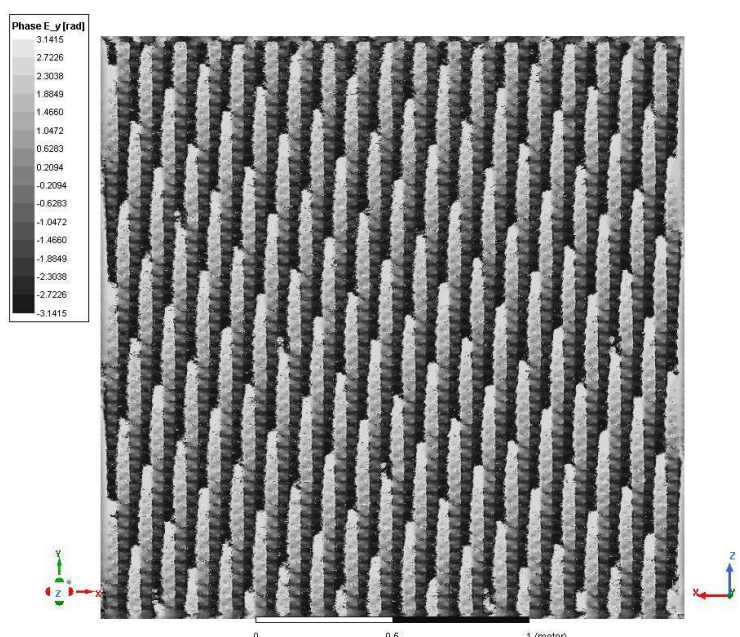

(d)

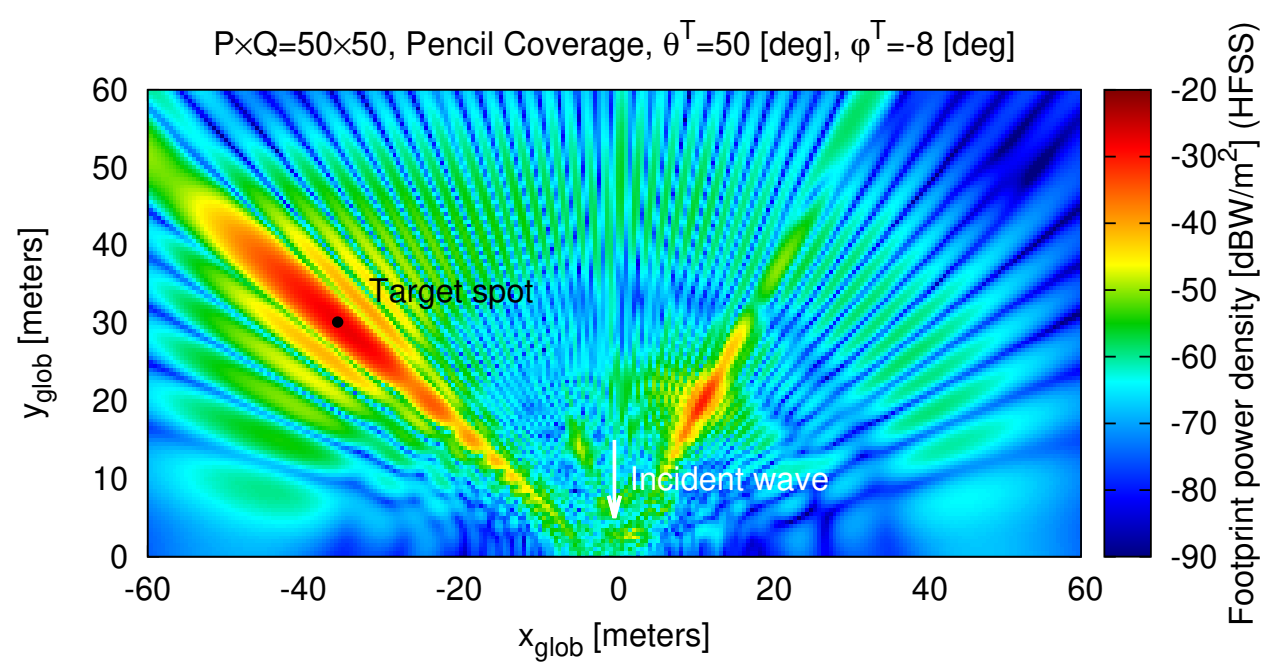

(b)

Fig. 9 - G. Oliveri et al., "Building a Smart EM Environment - AI-Enhanced Aperiodic ..." 


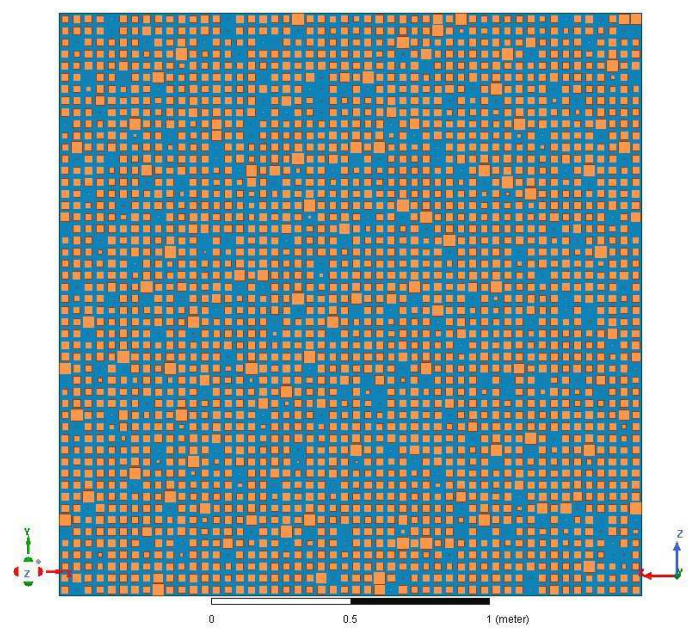

(a)

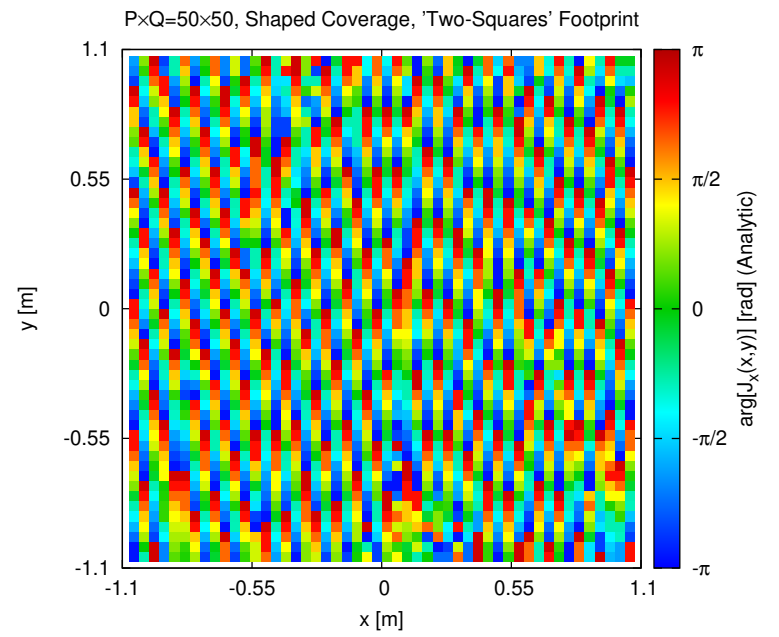

(c)

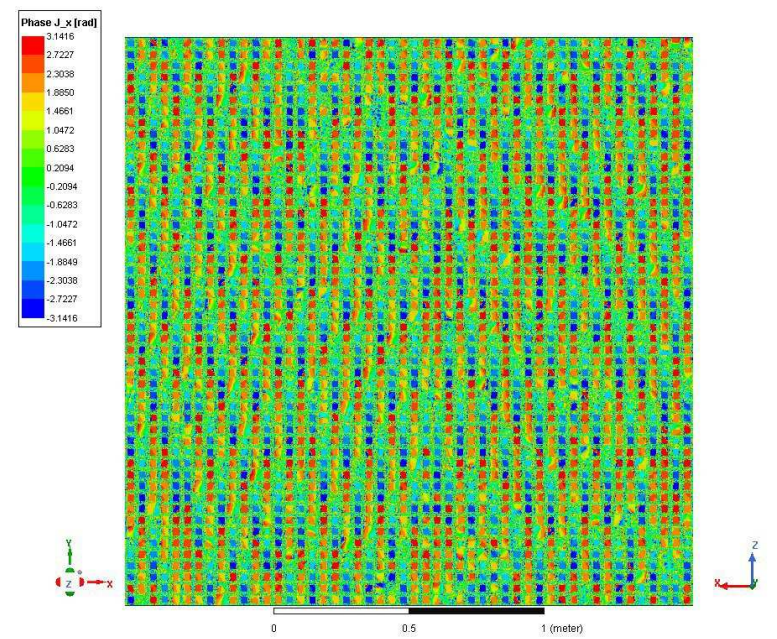

(e)

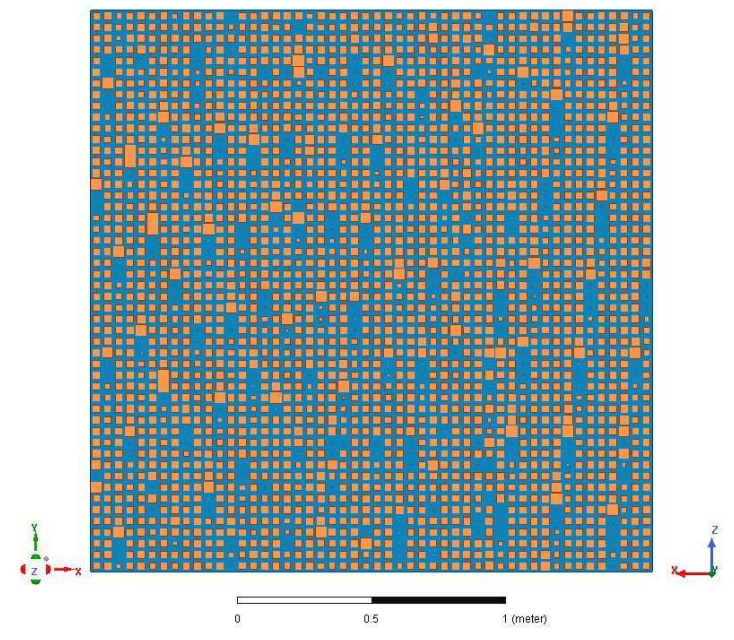

(b)

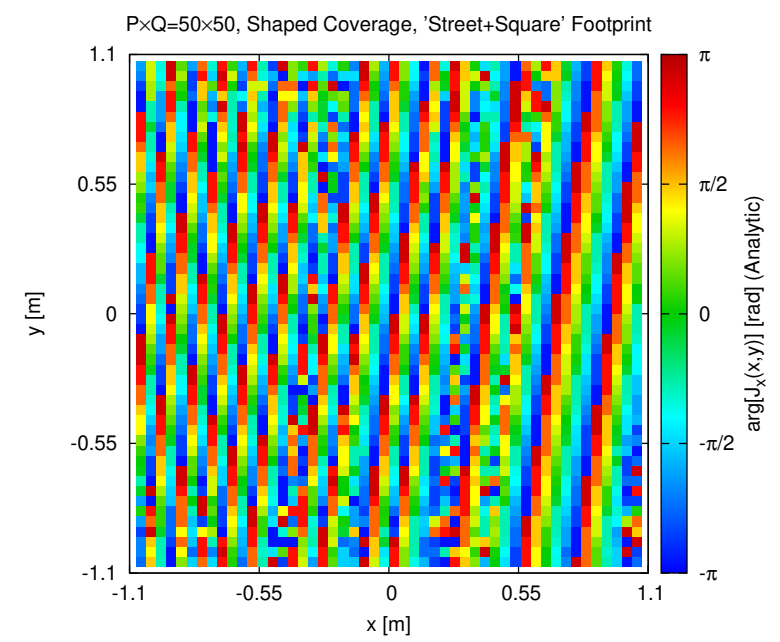

(d)

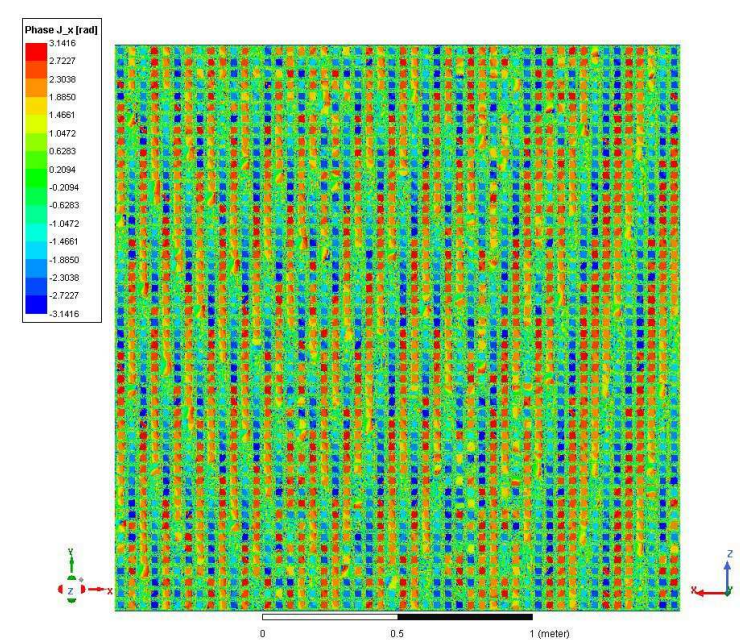

(f)

Fig. 10 - G. Oliveri et al., "Building a Smart EM Environment - AI-Enhanced Aperiodic ..." 


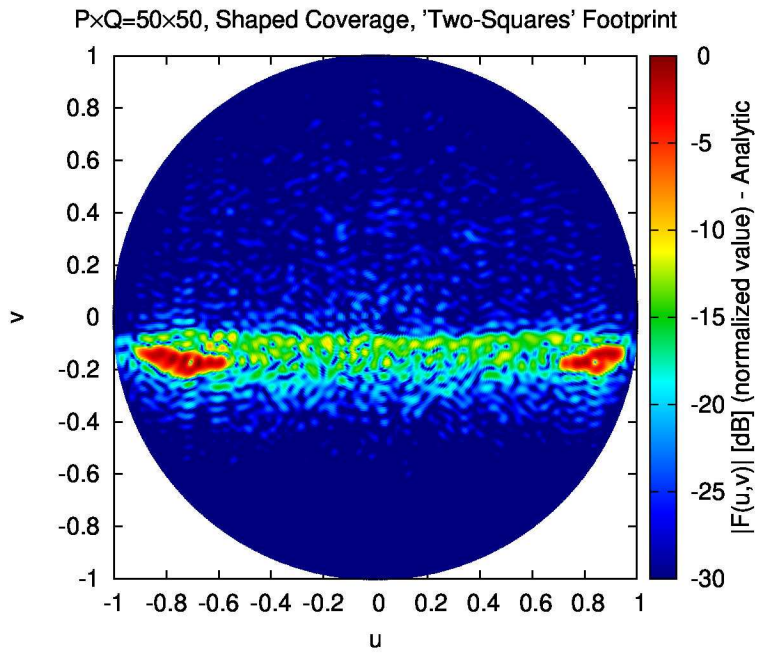

(a)

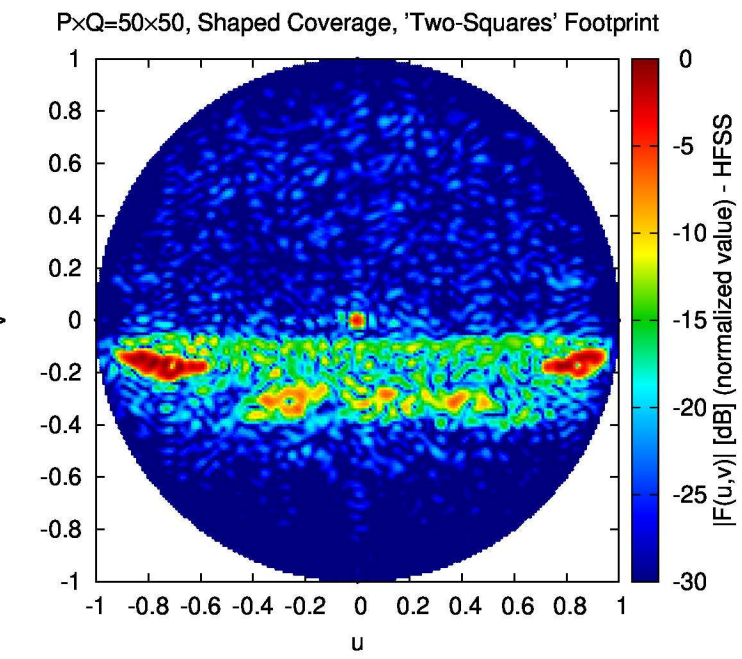

(c)
$P \times Q=50 \times 50$, Shaped Coverage, 'Street+Square' Footprint

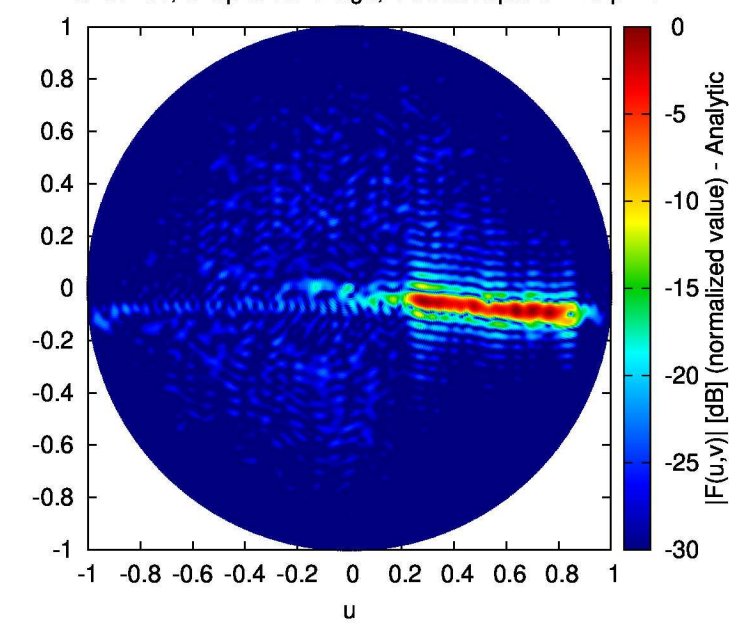

(b)

$P \times Q=50 \times 50$, Shaped Coverage, 'Street+Square' Footprint

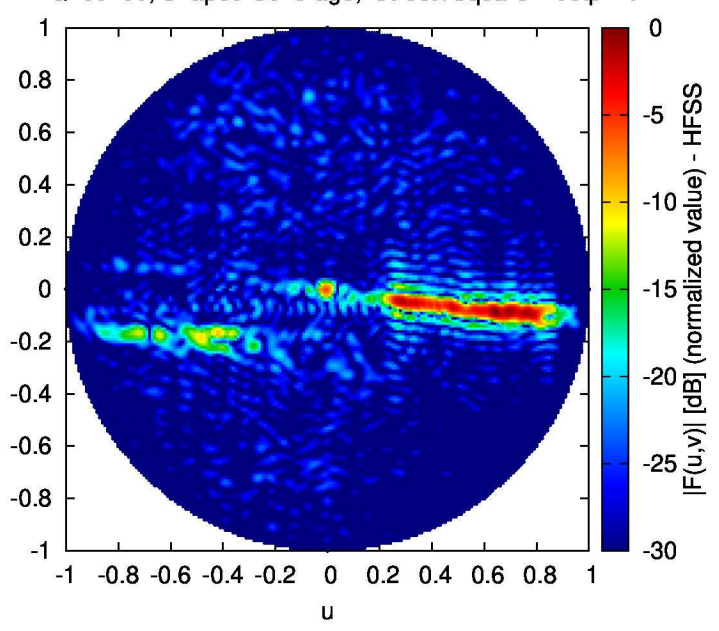

(d)

Fig. 11 - G. Oliveri et al., "Building a Smart EM Environment - AI-Enhanced Aperiodic ..." 


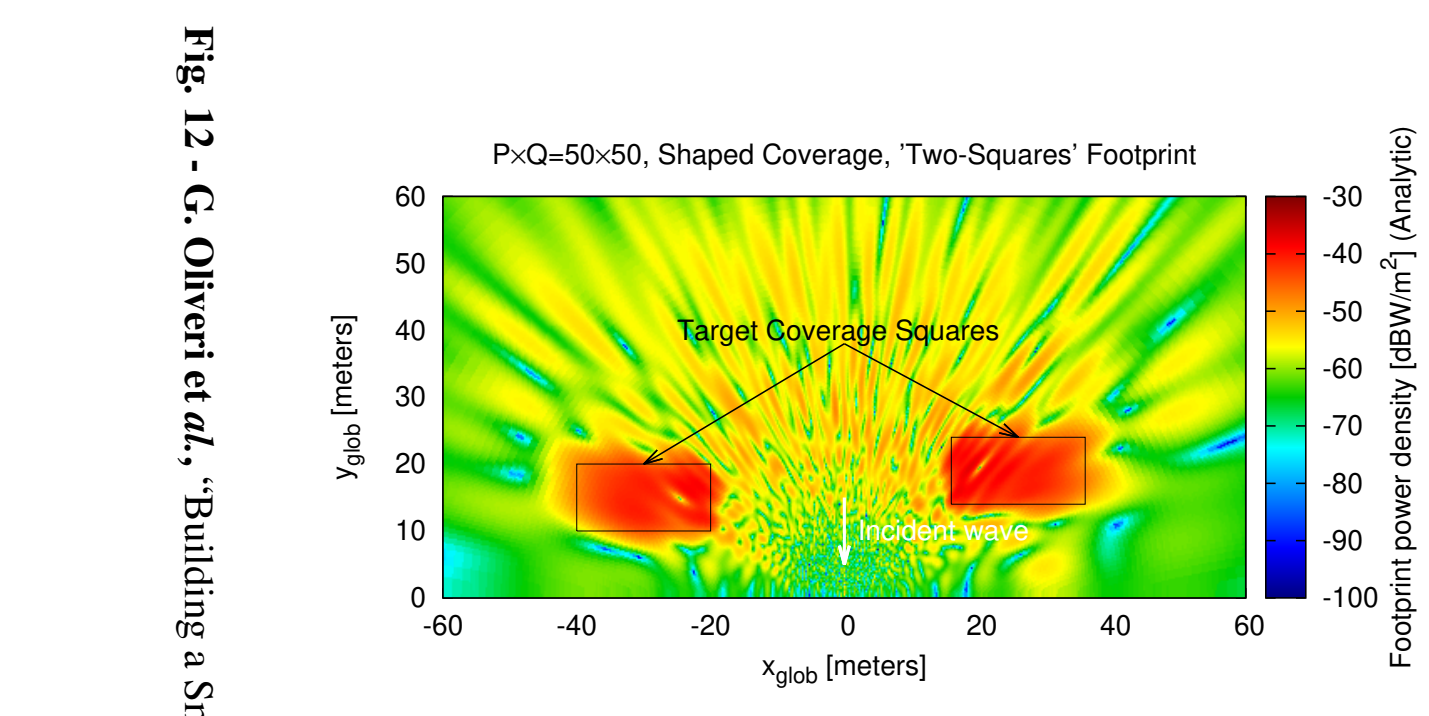

(a)

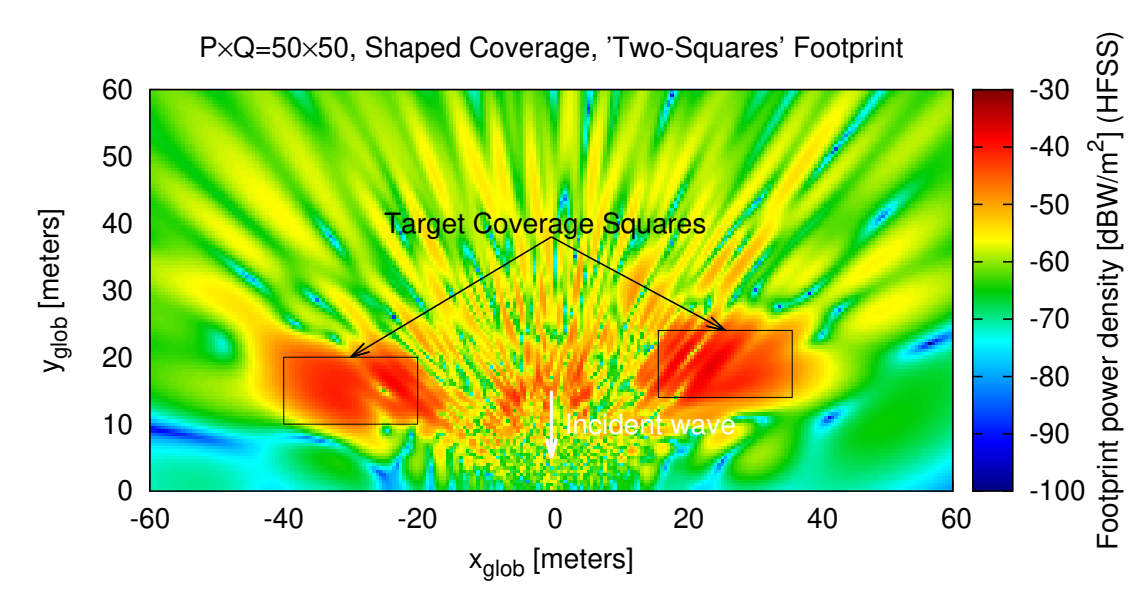

(c)

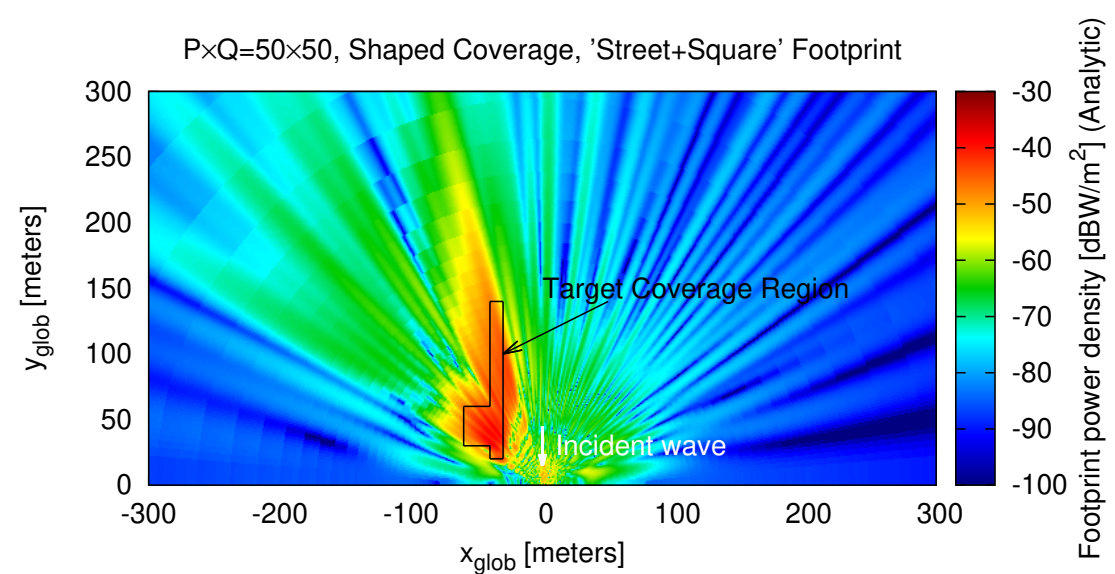

(b)

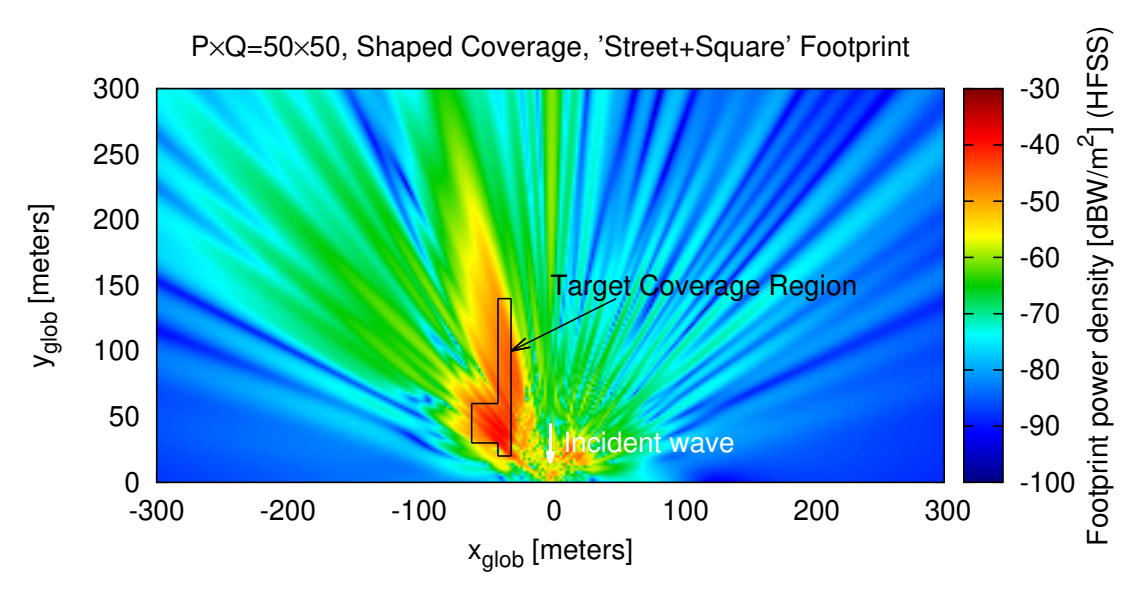

(d) 


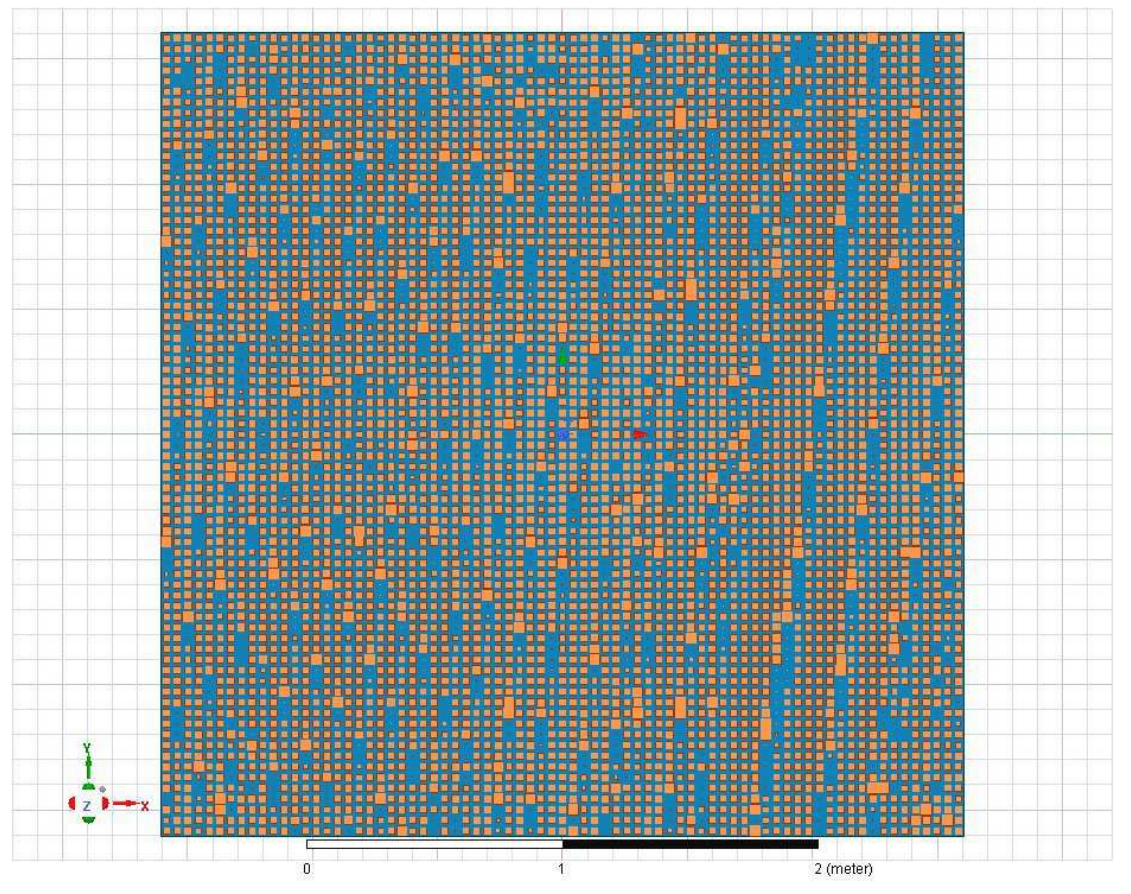

(a)

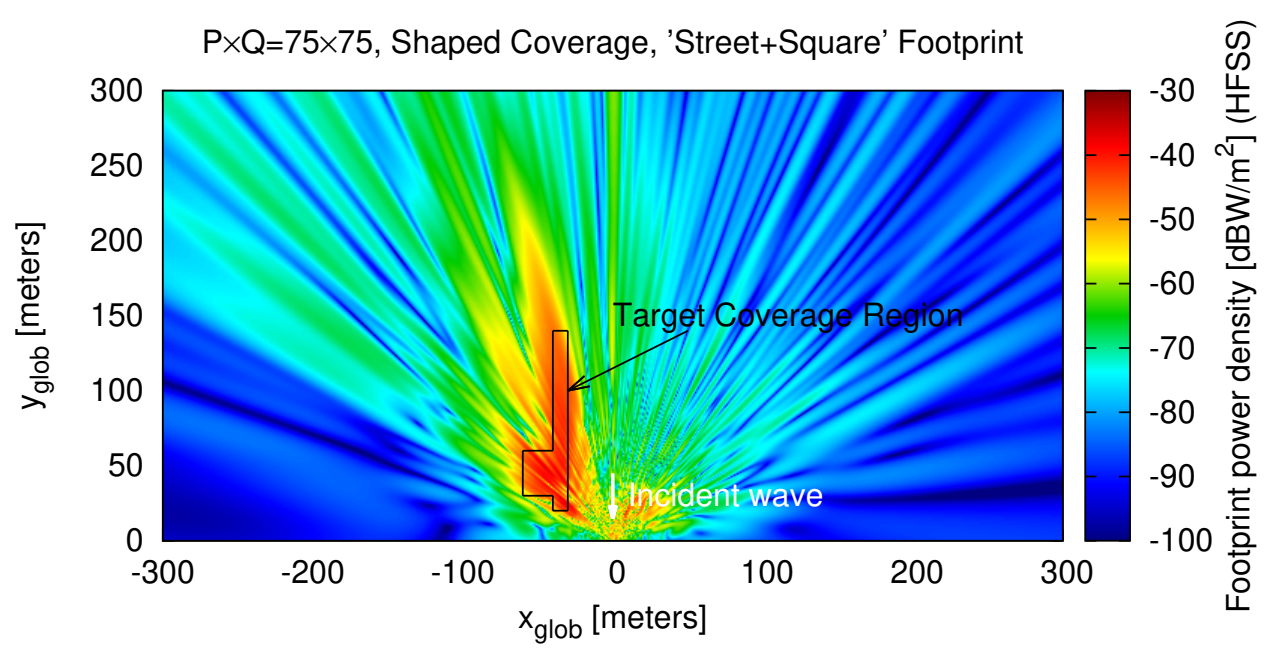

(b)

Fig. 13 - G. Oliveri et al., "Building a Smart EM Environment - AI-Enhanced Aperiodic ..." 


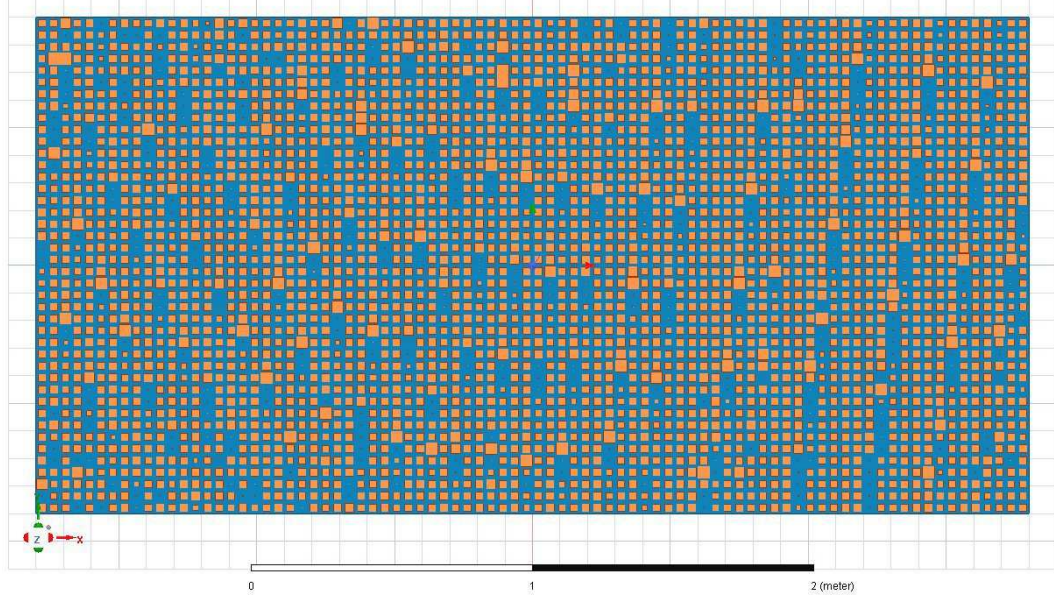

(a)

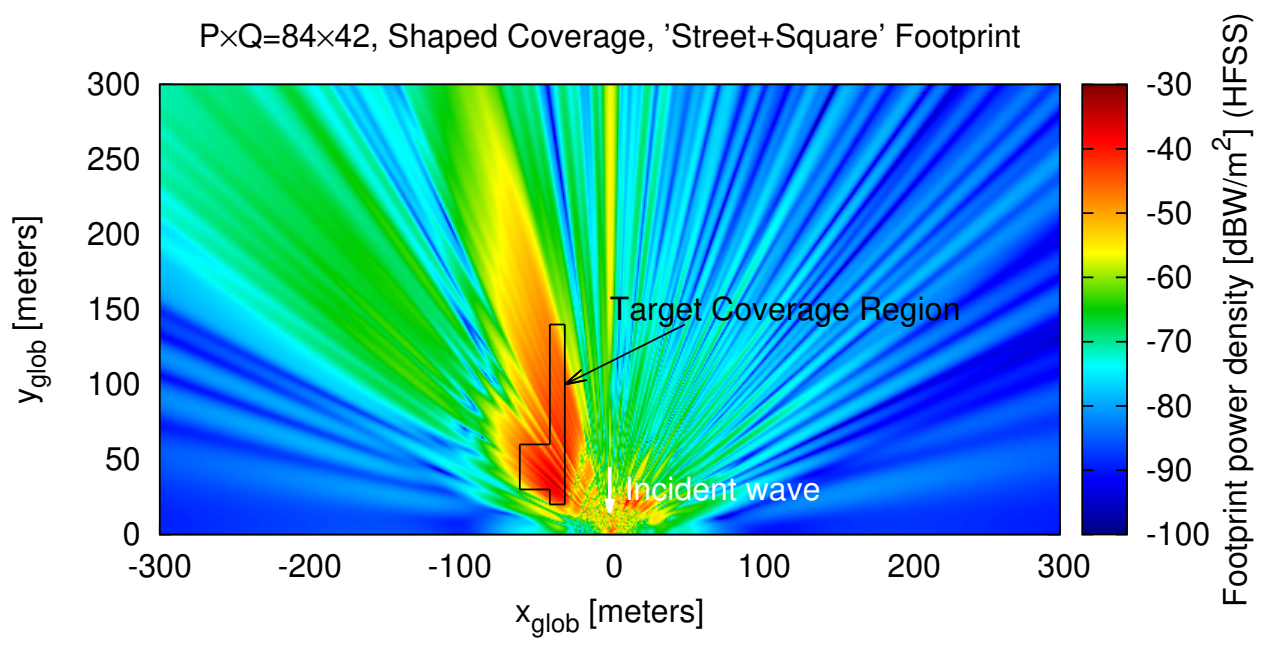

(b)

Fig. 14 - G. Oliveri et al., "Building a Smart EM Environment - AI-Enhanced Aperiodic ..." 


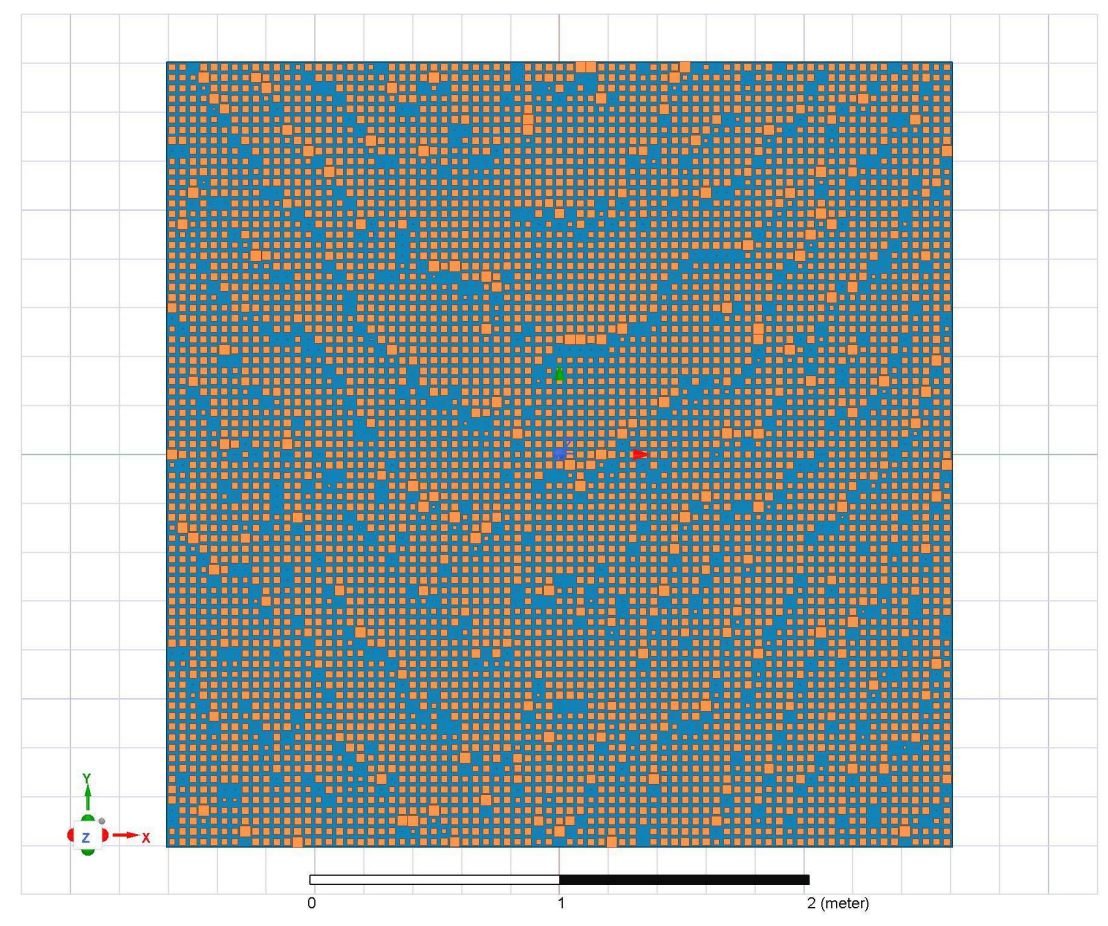

(a)

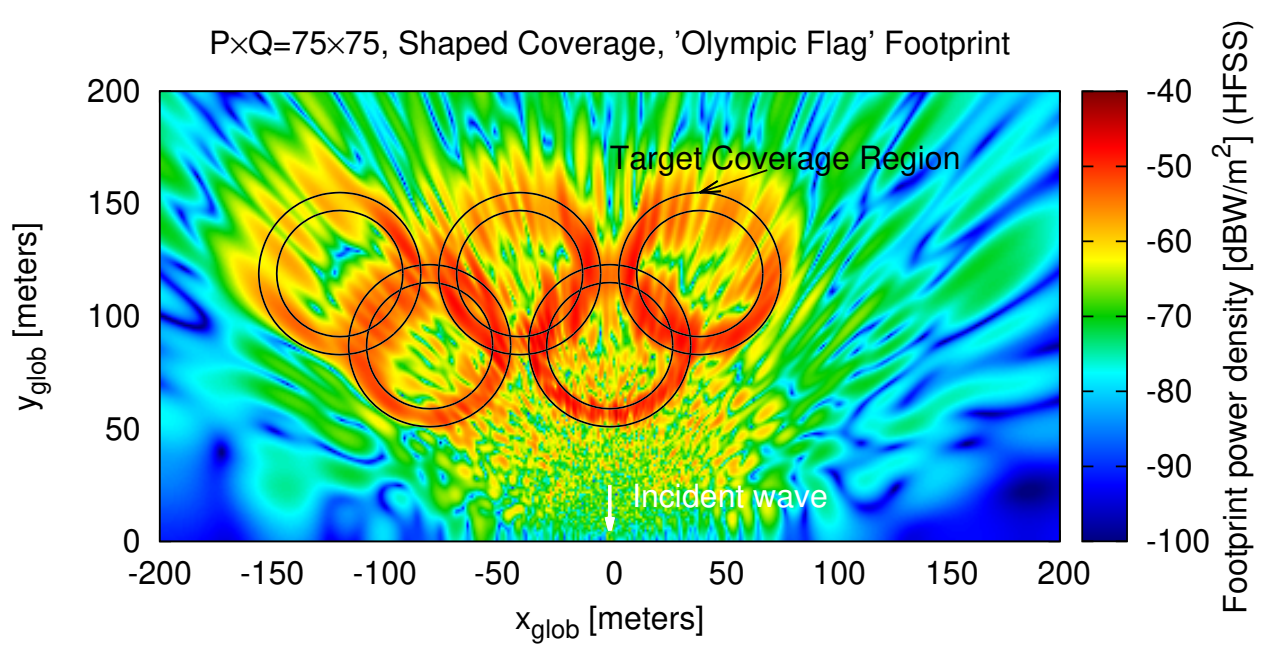

(b)

Fig. 15 - G. Oliveri et al., "Building a Smart EM Environment - AI-Enhanced Aperiodic ..." 